\title{
The Euler $T$ and Lambert $W$ functions in mechanistic radiobiological models with chemical kinetics for repair of irradiated cells
}

\author{
Dževad Belkić ${ }^{1,2}$
}

Received: 23 May 2018 / Accepted: 3 July 2018 / Published online: 10 July 2018

(c) The Author(s) 2018

\begin{abstract}
Recently, the Euler $T$ and Lambert $W$ transcendental functions found useful applications in cellular radiobiology. Specifically, these functions appeared in mathematical modeling of cell survival after irradiation using the formalism of chemical kinetics. An example is a mechanistic inclusion of cell repair through enzyme catalysis in the Michaelis-Menten formalism, where the concentration of radiation lesions is given by the Lambert function. This function also appears in cell surviving fractions in an alternative aspect of chemical kinetics when lesion repair is carried out by the so-called pool repair molecules without passing through enzyme catalysis. Moreover, even with no reference to chemical kinetics at all, both the Lambert and Euler functions emerge in the framework of the cell repair dynamics described by delayed differential equations. The Euler function is also encountered within the cell blocking mechanism of damage repair in analogy with the counting coincidence correction for the dead time of the radiation detecting instruments. Presently, we analyze the overall usefulness of the Lambert and Euler functions in radiotherapy with a goal of motivating a further exploration of this analytical methodology in mechanistic radiobiological models for cell survival. The main reason for pursuing this pathway is in its unified mechanistic concept of cell surviving fractions valid at all doses from low through intermediate to high radiation exposures. With this advance, no artificial cut-off doses are needed any longer for empirical connections of the intermediate and high dose regimens in mathematical modelings. This is particularly important in radiotherapy because the clinically most frequently used linear-quadratic model is inadequate at high doses. High doses are of main relevance to stereotactic radiotherapy for treatment of localized tumors by efficaciously administering relatively large doses per fraction in a small number of fractions within only a few days. Such a non-conventional treatment schedule is advantageous both for the patient and hospitals' cost effectiveness, especially relative to conventional radiotherapy, which uses small doses ( $2 \mathrm{~Gy}$ ) per fraction within a month long period.
\end{abstract}


Keywords Radiobiological models · Cell surviving fractions · Full-effect plots · Radiation damage repair · Michaelis-Menten enzyme catalysis · Pool molecules for repair

\section{Contents}

1 Introduction . . . . . . . . . . . . . . . . . . . . . . 2135

2 General usefulness of the Lambert $W$ and Euler $T$ functions . . . . . . . . . . . . . . . . . 2136

3 Direct functions and inverse functions . . . . . . . . . . . . . . . . . . . . . . . . . 2137

3.1 Direct functions . . . . . . . . . . . . . . . . . . . . . . . . . . . . . . . . . . . . . . . . . . . . . . . . . . . . . . .

3.2 Inverse functions . . . . . . . . . . . . . . . . . . . . . . . . 2138

3.3 Information-preserving and information-losing mappings . . . . . . . . . . . . . . 2138

4 The Euler $T(x)$ function . . . . . . . . . . . . . . . . . . . . . . . . . . . . . . . 2139

4.1 Inverse functions by reversion of the MacLaurin series . . . . . . . . . . . . . . . . . . 2139

4.2 Inverse functions by the Lagrange formula . . . . . . . . . . . . . . . . . . . . . . . . . 2141

4.3 The d'Alambert test ratio for the convergence radius . . . . . . . . . . . . . . . . . . . 2142

4.4 Definitions of the Euler $T$ function in terms of the linear-exponential and linear-logarithmic forms with no recourse to any series . . . . . . . . . . . . . . . . . . . . 2143

5 The Lambert $W(x)$ function . . . . . . . . . . . . . . . . . . . . . . . . . . . . . . . . . . . . 2144

5.1 Two branches $W_{0}$ and $W_{-1}$ of the multi-valued Lambert $W$ function . . . . . . . . . . . 2145

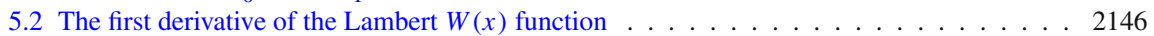

5.3 Indefinite integral of the Lambert $W(x)$ function . . . . . . . . . . . . . . . . . . . . . 2147

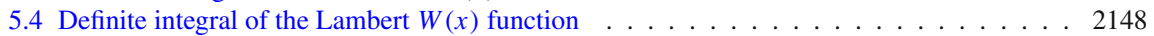

5.5 A first-order non-linear differential equation for the Lambert $W(x)$ function . . . . . . . . 2148

6 Compositional inverse for general functions . . . . . . . . . . . . . . . . . . . . . . 2148

6.1 Single-valued inverses . . . . . . . . . . . . . . . . . . . . . . . . . . . 2148

6.2 Multi-valued inverses . . . . . . . . . . . . . . . . . . . . 2150

7 Compositional inverses $T_{k}\left(x \mathrm{e}^{-x}\right)$ and $W_{k}\left(x \mathrm{e}^{x}\right)$ for $k=0$ and $k=-1 \ldots \ldots . \ldots 2151$

7.1 A key link between a branch choice and the independent variable domain . . . . . . . . 2151

7.2 Explicit proof of the compositional inverse for the Lambert function . . . . . . . . . . . . . 2154

8 Asymptotic behavior of $W(x)$ and $T(x)$ at small and large $x \ldots \ldots$. . . . . . . . . . . . . 2159

9 Applications of the Euler $T$ and Lambert $W$ functions in radiobiological modelings . . . . . . 2165

9.1 Integrated Michaelis-Menten method for cell survival . . . . . . . . . . . . . . . . . . . 2166

9.2 Cell repair by pool repair molecules from the cell environment . . . . . . . . . . . . . . . 2169

9.3 The Euler $T$ function for corrections to counting statistics . . . . . . . . . . . . . . . 2176

9.4 The Euler $T$ function stemming from counting statistics for cell survival . . . . . . . . . . 2177

9.5 The Euler $T$ function in cell survival stemming explicitly from delayed dynamics . . . . . 2178

9.6 The Euler $T$ function for cell survival based upon delayed dynamics and the Poisson dose modifying factor . . . . . . . . . . . . . . . . . . . . 2180

9.7 The Lambert $W$ function for cell survival based upon delayed dynamics . . . . . . . . . . 2181

10 Illustrations . . . . . . . . . . . . . . . . . . . . . . . . . . . 2182

11 Conclusions . . . . . . . . . . . . . . . . . . . . . . . 2185

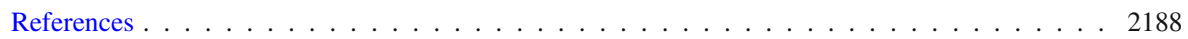
Abbreviations
BED Biologically effective dose
CF Continued fraction
DDE Delayed differential equation
DMF Dose modifying factor
DNA Deoxyribonucleic acid
EDD Euler delayed dynamics (model)
EPD Euler Poisson delayed (model) 
IMM Integrated Michaelis-Menten (model)

LDD Lambert delayed dynamics (model)

LQ Linear quadratic (model)

LQL Linear quadratic linear (model)

ODE Ordinary differential equation

PA Padé approximant

PRL Pool repair Lambert (model)

QSS Quasi stationary state

SBRT Stereotactic body radiation therapy

\section{Introduction}

In the present work, we review the recent advances in the field of radiobiological models [1-3] $(2014,2015)$. The main emphasis is placed onto survival of cells after exposure to radiation. This research theme, especially when implemented by nonconventional fractionated treatments with high doses, the stereotactic radiotherapy, is of direct relevance to radiotherapy in cancer medicine [4-13] (1951-2012). The reason is that eradication of cancerous cells is of utmost importance to the oncologist for fuller control of the disease and, ultimately, cure of patients with cancer.

The most frequently employed radiobiological model for conventional radiotherapy (2 Gy per fraction), called the linear-quadratic (LQ) model [14-23] (1938-2013) is inappropriate for stereotactic radiotherapy $[24,25](2008,2009)$. This occurs because at large values of dose $D$, the LQ model gives the quadratic $\left(D^{2}\right)$ dependence of the dose-effect relation, instead of the correct, linear $(D)$ behavior. Such a failure prompted researchers to use e.g. the Heaviside step function to link the low-dose LQ model with the proper linear high-dose asymptote of the dose-effect curve. One of such combinations is called the linear-quadratic-linear (LQL) model [26-36] (20042009). In the LQL model, the dose-effect curve is a discontinuous function of $D$ at a cut-off (or a transition dose) $D_{\mathrm{T}}$. Moreover, the LQL has no mechanistic basis similarly to the LQ model [21]. Further, the LQL model doubles the number of the adjustable parameters relative to the LQ model (4 vs. 2). This is one of the reasons for using certain alternatives as continuous, universal, cell survival curves that would be adequate at all doses and, thus, applicable to stereotactic radiotherapy, as well There exists a number of radiobiological models that can give such curves [37-53] (1963-2014).

We shall place the principal emphasis on the radiobiological models that use the Euler $T$ and Lambert $W$ functions for descriptions of cell repair within the system of coupled differential equations from chemical kinetics. Two such models based upon different repair mechanisms are the integrated Michaelis-Menten (IMM) [1] and the pool repair Lambert (PRL) [2] models. They both give the continuous cell surviving fractions at all doses, and predict the required asymptotes at small and large doses. As such they are optimal for dose planning systems in conventional and non-conventional radiotherapy at small and large doses per fraction, respectively. In the IMM and PRL models, the corresponding systems of the kinetic equations for the concentrations of radiation lesions, that yield the dose-effect curves, have previously been reduced 
to a linear-logarithmic [54,55] $(1971,1991)$, and a linear-exponential [56] (1972) transcendental equation.

However, the authors of Refs. [54-56] have not solved these latter equations by analytical means. Further, it has repeatedly been asserted [54,57,58] $(1985,1988)$ that such transcendental equations have no explicit, closed form solutions. It was not until recently [1,2] (2014) that the pertinent analytical solutions have been derived in terms of the Lambert $W$ function. This is how the IMM and PRL radiobiological models have emerged to advantageously facilitate both the analytical analyses and computations of dose-response curves covering smoothly all doses. These mechanistic models are computationally attractive because they each have only three radiobiologically interpretable parameters. Moreover, the open source codes, libraries and packages for the Lambert $W$ function can efficiently be employed with either high or unlimited accuracy.

The passage from low to high linear dose regions through the intermediate shouldered part of the dose-effect curve from the IMM and PRL models is automatically secured by the built-in Lambert $W$ function. This feature alone, in sharp contrast to Refs. [26-36], obviates the need for introducing a superficial transition dose $D_{\mathrm{T}}$ to force the logarithmic surviving fractions to exhibit linear dose behavior at high doses in an attempt to superficially enable an extension of the LQ model to stereotactic radiotherapy.

The plan of this presentation is as follows. In Sects. 2-8, we address, from an algebraic perspective, several issues relevant to the properties of the Euler $T$ and Lambert $W$ functions. Subsequently, in Sect. 9, we analyze the selected applications of these functions to radiobiological chemical kinetics with the focus on repair mechanisms for descriptions of the cell response to the imparted radiation. Section 10 deals with the numerical results for these representative illustrations concentrating especially on the relative performance of the LQ and IMM models. Finally, the conclusions regarding these applications are given in Sect. 11.

\section{General usefulness of the Lambert $W$ and Euler $T$ functions}

The Lambert $W$ [59,60] $(1758,1770)$ and Euler $T$ [61,62] $(1777,1783)$ functions are multi-valued inverses of (generally) complex linear-exponential functions of complex variables. For $W$, the corresponding direct function is $y=x \mathrm{e}^{x}$, so that $x=W(y)$. For $T$, we have $y=x \mathrm{e}^{-x}$ and, thus, $x=T(y)$. The relationship $T(y)=-W(-y)$ shows that neither function is even (symmetric) nor odd (anti-symmetric). Earlier, these functions have undergone several developmental stages through the articles of many authors, including Wright in the 1950s [63-65] (1949-1959), Siewert et al. in the 1970s [66-79] (1972-1979), Corless et al. in the 1990s [80-85] (1993-1999), Scott et al. [86,87] (1993), Schnell and Mendoza [88] (1997), Goudar et al. [89] (1999), etc.

Judging upon the abundant literature, prior to the first survey by Corless et al. [83] (1996) on this subject area, and after the recent reviews by Nastou et al. [90] (2016), Barsan [91] (2018) and an international workshop [92] (2016), a further significant progress is expected also in the future. This prospect is feasible thanks to a number of algorithms, libraries, packages (both numerical and symbolic) for computations 
of the $W$ and $T$ functions in several programing languages (Fortran, $\mathrm{C}++$, Matlab, Maple, Macsyma, Mathematica). Precision accuracy of the results ranges from high to unlimited, as provided by the codes of Fritsch et al. [93] (1973), Barry et al. [94] (1995), Bailey et al. [95,96] (2002, 2005), Gautschi [97,98] (2011), Veberič [99] (2012), Jeffrey et al. [100] (2015), Johansson [101] (2017), Adler [102] (2017), etc. Advantageously, some of the programs are available as open source codes.

The continued interest in the $W$ and $T$ functions across inter-disciplinary research is due to the fact that they are the exact, explicit, analytical solutions to many problems. The variety of the applications of these functions is dizzying in vastly different fields (mathematics, physics, chemistry, biology, medicine, ecology, sociology, education, agriculture, technology, engineering, etc). An extended and systematized (applicationwise) bibliography, partially covering all the mentioned areas can be found in Ref. [103] (2018).

\section{Direct functions and inverse functions}

\subsection{Direct functions}

The increasing and decreasing exponentials $\mathrm{e}^{x}$ and $\mathrm{e}^{-x}$, respectively, pre-multiplied by monomial $x$ are frequently used in mathematical modeling of various phenomena in biomedicine or ecology for species growth or decline, respectively:

$$
\begin{gathered}
y=x \mathrm{e}^{x}, \\
y=x \mathrm{e}^{-x} .
\end{gathered}
$$

Among the myriad of applications of functions of types (3.1) and (3.2), we could mention e.g. the Ricker model [104]:

$$
y=b x \mathrm{e}^{-a x}
$$

where $a$ and $b$ are positive constants. The Ricker model (3.3) for studies on populations of various species, including humans, is frequently used in ecology and environmental research [104]. The curve for $y$, as a function of the independent variable $x$, begins to grow linearly as $y \sim b x$ for small $x$ with the slope $b$, reaches its maximum at $x=1 / a$ and ends up by falling off exponentially via $y \sim \mathrm{e}^{-a x}$ at large values of $x$. Such a curve can describe the relationship between the size of the parental stock of some species $(x)$ and the number of recruits or off-springs $(y)$.

The validity of the Ricker model rests upon the assumption that, per capita, the ability to produce off-springs, i.e. fecundity, decreases exponentially with population density $x$. This is one possible mechanistic interpretation of the Ricker model within the context of population growth. In some other realms, the same function $y=b x \mathrm{e}^{-a x}$ could be interpreted as a phenomenological model for a dependent variable which first starts at zero, then increases to attain its peak value and finally afterward declines gradually back to zero. 


\subsection{Inverse functions}

Despite their widespread usefulness in applications, the simple functions (3.1) and (3.2) do not have their special names nor symbols in the mathematical literature. In contradistinction, however, the inverse functions $\left(x \mathrm{e}^{x}\right)^{(-1)}$ and $\left(x \mathrm{e}^{-x}\right)^{(-1)}$ of $x \mathrm{e}^{x}$ and $x \mathrm{e}^{-x}$ have their widely accepted names, suggested by Corless et al. [80,83], as the Lambert $W(x)$ and the Euler $T(x)$ functions, respectively:

$$
\begin{aligned}
& W(x)=\left(x \mathrm{e}^{x}\right)^{(-1)}, \\
& T(x)=\left(x \mathrm{e}^{-x}\right)^{(-1)} .
\end{aligned}
$$

We see from (3.4) and (3.5) that functions $W(x)$ and $T(x)$ are related to each other as:

$$
W(-x)=-T(x)
$$

This shows that neither the Lambert $W(x)$ nor the Euler $T(x)$ function is even (symmetric) nor odd (antisymmetric).

\subsection{Information-preserving and information-losing mappings}

Finding inverses $\left(x \mathrm{e}^{x}\right)^{(-1)}$ and $\left(x \mathrm{e}^{-x}\right)^{(-1)}$ could be meaningful only if the underlying mappings are injective. A function $F(x)$ is injective (one-to-one correspondence) whenever the relation $F\left(x_{1}\right)=F\left(x_{2}\right)$ implies $x_{1}=x_{2}$ and if, additionally, for $x_{1} \neq$ $x_{2}$, we have $F\left(x_{1}\right) \neq F\left(x_{2}\right)$ :

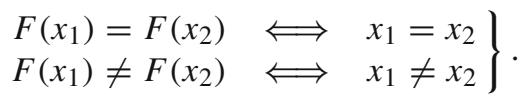

In other words, $F(x)$ would be injective if it maps a distinct object into another distinct object. It is said that an injective function preserves distinctiveness because it never maps distinct elements from its domain of definition to the same element of its image (co-domain), i.e. the relation $F\left(x_{1}\right)=F\left(x_{2}\right)$ is excluded for $x_{1} \neq x_{2}$. An injective function is information-preserving, whereas a non-injective function is informationlosing. Non-injection cannot be reversed to become injection, since it is impossible to obtain an information-preserving function from an information-losing function. Thus, strictly speaking a non-injective function $f(x)$ is not invertable, i.e. it has no inverse in the sense that $F^{(-1)}(x)$ does not exist. In such a case, we may speak of a pseudo- or quasi-inverse function $F^{(-1)}(x)$ as a multi-valued function. However, a multi-valued function $F(x)$ is not a proper or true function, since two or infinitely many values of $F(x)$ could be assigned to a single variable $x$.

This situation could be salvaged by separating different images $F(x)$ for the same $x$ into distinct branches $\left\{F_{0}(x), F_{ \pm 1}(x), F_{ \pm 2}(x), \ldots\right\}$ with each $F_{k}(x)(k=$ $0, \pm 1, \pm 2, \ldots)$ being a single-valued function, and where $k$ is the branch counting index. Then $f(x)$ is the union of all (possibly infinitely many) branches from the set 
$\left\{F_{0}(x), F_{ \pm 1}(x), F_{ \pm 2}(x), \ldots\right\}$. When both the dependent $(F(x))$ and independent $(x)$ variables are real, the injective function $F(x)$ can easily be visualized as the curve which is never crossed (intersected) more than once by any horizontal line (parallel with the abscissa $x$ ). This is called the horizontal test. For example, some horizontal lines could be chosen in such a way that they do not cross the functions $x \mathrm{e}^{x}$ and $x \mathrm{e}^{-x}$ plotted versus $x$. Therefore, these two latter functions are not injective and, hence, the related inverse (reverse) functions $\left(x \mathrm{e}^{x}\right)^{(-1)}$ and $\left(x \mathrm{e}^{-x}\right)^{(-1)}$ cannot uniquely be defined. Nevertheless, we can still speak of $\left(x \mathrm{e}^{x}\right)^{(-1)}$ and $\left(x \mathrm{e}^{-x}\right)^{(-1)}$ as being multivalued functions (with specified branches).

\section{The Euler $T(x)$ function}

\subsection{Inverse functions by reversion of the MacLaurin series}

We shall first find the inverse $\left(x \mathrm{e}^{-x}\right)^{(-1)}$ of function $x \mathrm{e}^{-x}$ from (3.2). The result was already mentioned in (3.5) by reference to the Euler $T(x)$ function via $T(x)=$ $\left(x \mathrm{e}^{-x}\right)^{(-1)}$. This will be established here by using the reversion of the MacLaurin power series expansion. To encompass both functions $x \mathrm{e}^{x}$ and $x \mathrm{e}^{-x}$ from (3.1) and (3.2), we will employ a slightly more general function of the type:

$$
y=x \mathrm{e}^{-a x},
$$

where the constant $a$ can be positive or negative. Finding the inverse function $\left(x \mathrm{e}^{-a x}\right)^{(-1)}$ amounts to obtaining all the real and complex roots $x$ of the transcendental equation $y=x \mathrm{e}^{-a x}$, where $y$ is known and $a$ is any given general constant (real or complex). Transcendental equations are alternatively called functional equations. Employing the MacLaurin series for $\mathrm{e}^{-a x}$, we have:

$$
y=\sum_{n=0}^{\infty} c_{n} x^{n}, \quad c_{n}=\frac{(-a)^{n}}{n !} .
$$

By reversion of this series, we will express $x$ as an expansion in powers of $y$ via:

$$
x=\sum_{n=1}^{\infty} b_{n} y^{n}, \quad b_{n}=\frac{1}{n !} x_{0}^{(n)},
$$

where $x_{0}^{(n)} \equiv\left\{x^{(n)}\right\}_{x=0}$ is the $n$th derivative of $x$ with respect to $y$ taken at $x=0$ with the standard notation:

$$
x^{(n)} \equiv \frac{\mathrm{d}^{n} x}{\mathrm{~d} y^{n}} .
$$

A recursion for calculating $x^{(n)}$ can be derived by first expressing $x^{(n)}$ as $(\mathrm{d} / \mathrm{d} y) x^{(n-1)}$ and then using the chain rule for the derivative $\mathrm{d} / \mathrm{d} y$ via $(\mathrm{d} / \mathrm{d} y)=(\mathrm{d} / \mathrm{d} x) /(\mathrm{d} y / \mathrm{d} x)=$ $\left[1 / y^{(1)}\right](\mathrm{d} / \mathrm{d} x)$ where $y^{(1)}=\mathrm{d} y / \mathrm{d} x$. This yields the sought recursion for $x^{(n)}$ : 


$$
x^{(n)}=\frac{1}{y^{(1)}} \frac{\mathrm{d}}{\mathrm{d} x} x^{(n-1)} .
$$

Denoting the $n$th derivative of $y$ with respect to $x$ by $y^{(n)}$ :

$$
y^{(n)} \equiv \frac{\mathrm{d}^{n} y}{\mathrm{~d} x^{n}}
$$

and employing (4.1), we obtain the closed formula for $y^{(n)}$ for any $n$ :

$$
y^{(n)}=(-a)^{n-1}(n-a x) \mathrm{e}^{-a x} .
$$

Using this expression in (4.4), the first few explicit derivatives $x^{(n)}$ can easily be found:

$$
\begin{aligned}
& x^{(1)}=\frac{\mathrm{e}^{a x}}{1-a x}, \\
& x^{(2)}=\left(2 a-a^{2} x\right) \frac{\mathrm{e}^{2 a x}}{(1-a x)^{3}}, \\
& x^{(3)}=\left(9 a^{2}-8 a^{3} x+2 a^{4} x^{2}\right) \frac{\mathrm{e}^{3 a x}}{(1-a x)^{5}}, \\
& x^{(4)}=\left(64 a^{3}-79 a^{4} x+36 a^{5} x^{2}-6 a^{6} x^{3}\right) \frac{\mathrm{e}^{4 a x}}{(1-a x)^{7}},
\end{aligned}
$$

which yields $x_{0}^{(1)}=1, x_{0}^{(2)}=2 a, x_{0}^{(3)}=9 a^{2}$ and $x_{0}^{(4)}=64 a^{3}$, so that:

$$
b_{1}=1, \quad b_{2}=\frac{2 a}{2 !}, \quad b_{3}=\frac{(3 a)^{2}}{3 !}, \quad b_{4}=\frac{(4 a)^{3}}{4 !}
$$

This permits deduction of the general expansion coefficient $b_{n}$ for any $n(0 \leq n \leq \infty)$ in the form:

$$
b_{n}=\frac{(n a)^{n-1}}{n !}
$$

Therefore, all the roots of Eq. (4.1) are given by the MacLaurin series:

$$
x=\sum_{n=1}^{\infty} \frac{(n a)^{n-1}}{n !} y^{n},
$$

which is equivalent to:

$$
x=\frac{1}{a}\left\{\sum_{n=1}^{\infty} \frac{(n)^{n-1}}{n !}(a y)^{n}\right\} .
$$


Here, the term in the curly brackets is identified as the Euler $T(a y)$ function and, thus, it follows from (4.11) that:

$$
x=\frac{1}{a} T(a y),
$$

with the definition

$$
\begin{aligned}
T(y) & \equiv \sum_{n=1}^{\infty} \frac{n^{n-1}}{n !} y^{n} \\
& =y+y^{2}+\frac{3}{2} y^{3}+\frac{8}{3} y^{4}+\frac{125}{24} y^{5}+\frac{54}{5} y^{6}+\frac{16807}{720} y^{7}+\cdots
\end{aligned}
$$

\subsection{Inverse functions by the Lagrange formula}

As an alternative to Sect. 4.1, we shall use here the Lagrange inversion theorem. To this end, we assume that we are given a dependence between $x$ and $y$ in the implicit form:

$$
f(x)=y,
$$

where $f$ is the known function which is analytic at a point $x=a$ where $[(\mathrm{d} / \mathrm{d} x) f(x)]_{x=a} \neq 0$. Then, the Lagrange theorem yields the inverse function $f^{(-1)} \equiv g$ with:

$$
x=g(y) \text {, }
$$

where $g$ is analytic at the point $b \equiv f(a)$. The functional form of $g(y)$ is given by its Taylor series expansion in powers of $y-b$ :

$$
g(y)=a+\sum_{n=1}^{\infty}\left\{\frac{\mathrm{d}^{n-1}}{\mathrm{~d} x^{n-1}}\left(\frac{x-a}{f(x)-b}\right)^{n}\right\}_{x=a} \frac{(y-b)^{n}}{n !} .
$$

In the special case $a=0=b$, it is seen that (4.17) becomes the corresponding MacLaurin series:

$$
g(y)=\sum_{n=1}^{\infty}\left\{\frac{\mathrm{d}^{n-1}}{\mathrm{~d} x^{n-1}}\left(\frac{x}{f(x)}\right)^{n}\right\}_{x=0} \frac{y^{n}}{n !} .
$$

If we choose $f(x)$ to be of the form (4.1) via $f(x)=x \mathrm{e}^{-a x}$, then for $x / f(x)=\mathrm{e}^{a x}$ it follows:

$$
g(y)=\sum_{n=1}^{\infty}\left\{\frac{\mathrm{d}^{n-1}}{\mathrm{~d} x^{n-1}} \mathrm{e}^{\text {nax }}\right\}_{x=0} \frac{y^{n}}{n !} .
$$


The $(n-1)$ st derivative of $\mathrm{e}^{\text {nax }}$ with respect to $x$ is equal to $(a n)^{n-1} \mathrm{e}^{\text {nax }}$ which becomes $(a n)^{n-1}$ at $x=0$. Inserting this result into (4.19) and accounting for (4.16), we finally obtain:

$$
g(y)=x=\sum_{n=1}^{\infty} \frac{(a n)^{n-1}}{n !} y^{n}
$$

in agreement with (4.11). This, in turn, yields:

$$
g(y)=x=\frac{1}{a} T(a y),
$$

which coincides with (4.13). Overall, the two different methods, i.e. reversion of the MacLaurin series, and the Lagrange inversion formula give the same result via (4.13) and (4.21), respectively.

\subsection{The d'Alambert test ratio for the convergence radius}

In order to establish convergence of the series (4.11), we rewrite it as:

$$
x=\sum_{n=1}^{\infty} C_{n}, \quad C_{n}=\frac{(n a)^{n-1}}{n !} y^{n},
$$

and apply the d'Alambert ratio test. According to this test:

$$
\lim _{n \rightarrow \infty}\left|\frac{C_{n+1}}{C_{n}}\right|=q
$$

the series (4.22) is absolutely convergent provided that $q<1$. We calculate:

$$
q=\lim _{n \rightarrow \infty}\left|\frac{C_{n+1}}{C_{n}}\right|=\lim _{n \rightarrow \infty} \mid \frac{n}{n+1}\left(1+\frac{1}{n}\right)^{n} \text { ay }|=\mathrm{e}| a y \mid,
$$

where by definition, $\lim _{n \rightarrow \infty}(1+1 / n)^{n}=\mathrm{e}(\mathrm{e} \approx 2.71828)$. Hence, the radius $\rho_{\mathrm{c}}$ of convergence of series (4.11) is equal to $1 /(\mathrm{e}|a|)$ :

$$
|y|<\rho_{\mathrm{c}}, \quad \rho_{\mathrm{c}}=\frac{1}{\mathrm{e}|a|} \approx \frac{0.36787}{|a|} .
$$

Thus, the expansion (4.11) is valid in the disc of radius $\rho_{\mathrm{c}}$ where $\rho_{\mathrm{c}}=\{y:|y|<$ $1 /(\mathrm{e}|a|)\}$ :

$$
x=\sum_{n=1}^{\infty} \frac{(n a)^{n-1}}{n !} y^{n}, \quad \text { Convergence radius : } \rho_{\mathrm{c}}=\left\{y:|y|<\frac{1}{\mathrm{e}|a|}\right\} .
$$


In fact, the convergence radius $\rho_{\mathrm{c}}$ can be enlarged to become $\tilde{\rho}_{\mathrm{c}}$, which includes the point $|y|=1 /(\mathrm{e}|a|)$. This can be shown as follows. Substituting $1 /(\mathrm{e} a)$ for $y$ into $C_{n} \equiv C_{n}(y)$, we shall have $C_{n}(1 /\{\mathrm{e} a\})=(a n)^{n-1} \mathrm{e}^{-n} / n$ ! where $n$ ! can be replaced by the leading term of the Stirling series [105] via $n !=\sqrt{2 \pi} n^{n+1 / 2} \mathrm{e}^{-n}[1+1 /(12 n)+$ $\left.1 /\left(288 n^{2}\right)+\cdots\right]$ to give $C_{n} \approx a^{n-1} /\left(\sqrt{2 \pi} n^{3 / 2}\right)$. This specifies (4.11) as:

$$
x \approx \sum_{n=1}^{\infty} \tilde{C}_{n}, \quad \tilde{C}_{n}=\frac{a^{n-1}}{\sqrt{2 \pi} n^{3 / 2}} \quad \text { for } \quad y=\frac{1}{\mathrm{e} a}
$$

Note that for $a=1$ this series is proportional to the Riemann zeta function $\sum_{n=1}^{\infty} n^{-3 / 2}=\zeta(3 / 2) \approx 2.612$, so that $x \approx(2 \pi)^{-1 / 2} \sum_{n=1}^{\infty} n^{-3 / 2} \approx 2.612 / \sqrt{2 \pi} \approx$ 1.042. The ratio test on the series in (4.26) implies:

$$
\tilde{q}=\lim _{n \rightarrow \infty}\left|\frac{\tilde{C}_{n+1}}{\tilde{C}_{n}}\right|=\frac{1}{\sqrt{2 \pi}} \lim _{n \rightarrow \infty}\left|a\left(1+\frac{1}{n}\right)^{3 / 2}\right|=\frac{|a|}{\sqrt{2 \pi}} .
$$

Therefore, the series (4.26) converges absolutely for $\tilde{q}<1$ with the convergence radius equal to $(1 /|a|) \sqrt{2 \pi}$. This implies that the series (4.11) is well defined at $y=1 /(\mathrm{e} a)$ and, therefore, the new convergence radius $\tilde{\rho}_{\mathrm{c}}$ of this series is larger than $\rho_{\mathrm{c}}$ from (4.25), i.e. $\tilde{\rho}_{\mathrm{c}}>\rho_{\mathrm{c}}$ :

$$
x=\sum_{n=1}^{\infty} \frac{(n a)^{n-1}}{n !} y^{n}, \quad \text { Convergence radius : } \tilde{\rho}_{\mathrm{c}}=\left\{y:|y| \leq \frac{1}{\mathrm{e}|a|}\right\} .
$$

\subsection{Definitions of the Euler $T$ function in terms of the linear-exponential and linear-logarithmic forms with no recourse to any series}

We saw that the inverse $\left(x \mathrm{e}^{-a x}\right)^{(-1)}$ of function $y=x \mathrm{e}^{-a x}$ is equal to $(1 / a) T(a y)$. Likewise, the inverse $\left(b x \mathrm{e}^{-a x}\right)^{(-1)}$ of a slightly more general function given by the Ricker model $y=b x \mathrm{e}^{-a x}$ from (3.3), rewritten as $(a / b) y=a x \mathrm{e}^{-a x}$, is deduced as:

$$
\left(b x \mathrm{e}^{-a x}\right)^{(-1)}=\frac{1}{a} T\left(\frac{a}{b} y\right) .
$$

For the simpler case $a=1=b$ encountered in (3.2), the relation (4.29) becomes:

$$
\left(x \mathrm{e}^{-x}\right)^{(-1)}=T(x)=\sum_{n=1}^{\infty} n^{n-1} \frac{x^{n}}{n !}
$$

as announced in (3.5). In enumerative combinatorics [106,107], the Euler $T(x)$ function is used frequently under the name 'the tree function' (or the rooted tree function), with the alternative notation $R(x)$, which is written as: 


$$
R(x)=\sum_{n=1}^{\infty} r(n) \frac{x^{n}}{n !}, \quad r(n) \equiv n^{n-1}, \quad R(x)=T(x) .
$$

Here, the term $r(n) \equiv n^{n-1}$ represents the number of the so-called rooted trees on $n$ vertices. A set whose every connected component is a rooted tree is called a forest of rooted trees (or a rooted forest, or a planted forest). If $p_{k}(n)$ denotes the total number of planted forests with $k$ components on the vertex set $\{n\}$, then the factor $r(n)$ from (4.31) is given by $r(n)=p_{1}(n)$ [107].

The power series expansion (4.14) is only one of the representations of the Euler $T$ function. Another, more generic definition of the $T$ function can be established with no reference whatsoever to any power series expansion. To this end, we start from the fact that for a known $y$, the solution of the transcendental equation $y=x \mathrm{e}^{-x}$ is $x=T(y)$ :

$$
y=x \mathrm{e}^{-x} \quad \therefore \quad x=T(y) .
$$

Here, a simple replacement of $x$ by $T(y)$ permits re-writing (4.32) as:

$$
y=T(y) \mathrm{e}^{-T(y)} .
$$

This relation can serve as another definition of the Euler $T(y)$ function with no recourse to power series expansions. An alternative defining relation for $T(y)$ can be introduced by taking the natural (Naperian) logarithm of both sides of Eq. (4.33) for real $y$ :

$$
\ln T(y)-T(y)=\ln y .
$$

\section{The Lambert $W(x)$ function}

For a given $y$, the solution of the transcendental equation $y=x \mathrm{e}^{x}$ is $x=W(y)$ :

$$
y=x \mathrm{e}^{x} \quad \therefore \quad x=W(y) .
$$

Therefore, if we substitute $W(y)$ for $x$ into the growth function $y=x \mathrm{e}^{x}$ from (3.1), it would follow:

$$
y=W(y) \mathrm{e}^{W(y)} .
$$

This is one of the general defining relations of the Lambert $W(y)$ function. Further, with the natural logarithm taken of both sides of Eq. (5.2), an equivalent definitions of the Lambert $W$ function can be deduced as:

$$
\ln W(y)+W(y)=\ln y .
$$


According to (3.6) and (4.14), it is possible to write the power series representation of the Lambert $W(y)$ function in the form:

$$
\begin{aligned}
W(y) & \equiv \sum_{n=1}^{\infty} \frac{(-n)^{n-1}}{n !} y^{n} \\
& =y-y^{2}+\frac{3}{2} y^{3}-\frac{8}{3} y^{4}+\frac{125}{24} y^{5}-\frac{54}{5} y^{6}+\frac{16807}{720} y^{7}-\cdots
\end{aligned}
$$

Comparing the Euler $T(y)$ and the Lambert $W(y)$ functions from (4.14) and (5.4), respectively, the following general relationship is found for any $y$ :

$$
T(y)=-W(-y) .
$$

This is known already from the definitions (3.4) and (3.5) of the inverses of the growth and decline functions, respectively, as stated in (3.6). The same connection (5.5) is also implied by (4.33) and (5.2) or by (4.34) and (5.3).

\subsection{Two branches $W_{0}$ and $W_{-1}$ of the multi-valued Lambert $W$ function}

As we saw, the Lambert $W$ function, conceived through the relation $x=W(y)$ from (5.1), is the multi-valued solution of the implicit, transcendental equation $y=x \mathrm{e}^{x}$. The multi-valuedness of $W$, as indicated by writing $W_{k}$ instead of $W$ with $k=0, \pm 1, \pm 2, \ldots$, stems from its multiple branches that correspond to multiple roots of Eq. (5.1). In particular, $W_{0}(x)$ is the principal branch of $W(x)$. For real $x$, from the whole set $\left\{W_{k}(x)\right\}(k=0, \pm 1, \pm 2, \pm 3, \ldots)$ of the solutions of Eq. (5.1), only $W_{0}(x)$ and $W_{-1}(x)$ are real and single-valued functions.

All the other branches $\left\{W_{k}(x)\right\}(k=1, \pm 2, \pm 3, \ldots)$ are complex and multivalued irrespective of whether $x$ is real or complex. Moreover, $W_{0}(x)$ and $W_{-1}(x)$ are real-valued only in certain restricted $x$-intervals. Specifically, the real values of the single-valued $W_{0}(x)$ function are located in the interval $x \in[-1 / \mathrm{e},+\infty)$. On the other hand, the real, single-valued function $W_{-1}(x)$ lies in the interval $x \in[-1 / \mathrm{e}, 0)$. Outside the said intervals, these two branches of $W(x)$ are complex-valued even for real $x$. In other words, if we relax the restriction to real-valuedness of $W(x)$, the domain of the definition of both $W_{0}(x)$ and $W_{-1}(x)$ could be extended to encompass the whole real axis $x$. However, real-valuedness of $W(x)$ is not the only criterion for obtaining the unique real roots of Eq. (5.1). This is the case because $W(x)$ has two values for every $x \in[-1 / \mathrm{e}, 0]$, except for the branch point at $x=-1 / \mathrm{e}$, where:

$$
W_{0}(-1 / \mathrm{e})=1=W_{-1}(-1 / \mathrm{e})
$$

Moreover, near the essential singularity $x=-1 / \mathrm{e}$, we have:

$$
\left.\begin{array}{c}
W_{0}(x)_{x \rightarrow-1 / \mathrm{e}}-1+u \\
W_{-1}(x)_{x \rightarrow-1 / \mathrm{e}}-1-u
\end{array}\right\},
$$


with

$$
u=\sqrt{2(1+\mathrm{e} x)} .
$$

Thus, to secure uniqueness of the real solution $x$ of Eq. (5.1) for $x \geq-1 / \mathrm{e}$, the function $W(x)$ must be split into two branches each of which is single-valued. This is achieved by subdividing the images $W(x)$ into two sets $W(x) \geq-1$ and $W(x) \leq-1$ :

$$
W(x)= \begin{cases}W_{0}(x), & W(x) \geq-1 \\ W_{-1}(x), & W(x) \leq-1\end{cases}
$$

or equivalently

$$
W(x)= \begin{cases}W_{0}(x), & x \in[-1 / \mathrm{e},+\infty) \\ W_{-1}(x), & x \in[-1 / \mathrm{e}, 0] .\end{cases}
$$

Using the relationship $-W(-x)=T(x)$ from (3.6) or (5.5), we can also deduce a similar specification for the two branches $T_{0}(x)$ and $T_{-1}(x)$ of the Euler $T$ function function:

$$
T(x)= \begin{cases}T_{0}(x), & T(x) \leq 1 \\ T_{-1}(x), & T(x) \geq 1\end{cases}
$$

Most applications of the Lambert function employ the branches $W_{0}(x), W_{-1}(x)$ and $W_{1}(x)[83,93,97,98,108,109]$. We shall also analyze the $T$ and $W$ functions with the particular arguments $x \mathrm{e}^{-x}$ and $x \mathrm{e}^{x}$, respectively, namely $T_{k}\left(x \mathrm{e}^{-x}\right)$ and $W_{k}\left(x \mathrm{e}^{x}\right)$. Generally, these special values of the $T$ and $W$ functions are not equal to $x$ and, therefore, they are denoted by $\widetilde{T}_{k}$ and $\widetilde{W}_{k}$, respectively:

$$
\begin{aligned}
\widetilde{T}_{k} & \equiv T_{k}\left(x \mathrm{e}^{-x}\right), \\
\widetilde{W}_{k} & \equiv W_{k}\left(x \mathrm{e}^{x}\right) .
\end{aligned}
$$

\subsection{The first derivative of the Lambert $W(x)$ function}

Application of the operator $\mathrm{d} / \mathrm{d} x$ to both sides of Eq. (5.2) yields the following rule for the first derivative of the Lambert $W$ function:

$$
\frac{\mathrm{d} W(x)}{\mathrm{d} x}=\frac{\mathrm{e}^{-W(x)}}{1+W(x)}, \quad x \neq-\frac{1}{\mathrm{e}} .
$$

As seen, function $W(x)$ is not differentiable at $x=-1 /$ e due to singularity $W^{\prime}(-1 / \mathrm{e})=\infty$ of $W^{\prime}(x) \equiv \mathrm{d} W(x) / \mathrm{d} x$. By contrast, according to (5.6), functions $W_{0}(x)$ and $W_{-1}(x)$ are well defined at the essential singularity point, $x=-1 / \mathrm{e}$, at 
which $W_{0}(-1 / \mathrm{e})=W_{-1}(-1 / \mathrm{e})=1$. Using the definition $(5.2)$ to replace $\mathrm{e}^{-W(x)}$ by $x^{-1} W(x)$ in (5.3), the following equivalent expression is obtained for the derivative:

$$
\frac{\mathrm{d} W(x)}{\mathrm{d} x}=\frac{W(x)}{x\{1+W(x)\}}, \quad x \neq 0, \quad x \neq-\frac{1}{\mathrm{e}} .
$$

The higher-order derivatives $(\mathrm{d} / \mathrm{d} x)^{n} W(x)$ can be generated from (5.14) in terms of the Eulerian polynomials of the second kind [80].

\subsection{Indefinite integral of the Lambert $W(x)$ function}

It is also possible to readily calculate certain indefinite integrals (i.e. the so-called primitive functions) containing $W(x)$, such as:

$$
I \equiv \int W(x) \mathrm{d} x
$$

Here, in view of the defining relation (5.2), we change the integration variable from $x$ to $W(x)$ according to:

$$
x=W(x) \mathrm{e}^{W(x)} \quad \therefore \quad \mathrm{d} x=\{1+W(x)\} \mathrm{e}^{W(x)} \mathrm{d} W(x) .
$$

This maps (5.16) into an elementary integral in which $W(x)$ becomes the integration variable:

$$
I=\int W(x)\{1+W(x)\} \mathrm{e}^{W(x)} \mathrm{d} W(x)
$$

so that

$$
I=x\left\{W(x)-1+\frac{1}{W(x)}\right\}+C_{I}, \quad x \neq 0,
$$

where $C_{I}$ is the integration constant. Similarly, employing (5.17), it is also easy to obtain the primitive function $J$ of the integrand $(1 / x) W(x)$ :

$$
J \equiv \int \frac{W(x)}{x} \mathrm{~d} x
$$

Thus, referring again to $(5.2)$ in the form $(1 / x) W(x)=\mathrm{e}^{-W(x)}$, and taking into account (5.17), we have from (5.20):

$$
J=\int\{1+W(x)\} d W(x)=W(x)+\frac{1}{2} W^{2}(x)+C_{J},
$$

with $C_{J}$ being the constant of integration. With the same variable change and its differential from (5.17), many other indefinite integrals $\int \mathrm{d} x f(x, W(x))$ could likewise 
be analytically calculated with the integrand $f(x, W(x))$ containing various functional forms of $x$ and $W(x)$ [80].

\subsection{Definite integral of the Lambert $W(x)$ function}

A number of definite integrals has also been considered in the literature. For example, Gautschi $[97,98]$ has recently studied the following integrals with the real-valued integrands:

$$
\begin{aligned}
I_{\mathrm{G}} & \equiv \int_{1}^{\infty} x^{-\beta}\left\{T_{0}\left(x \mathrm{e}^{-x}\right)\right\}^{\alpha} \mathrm{d} x, \\
J_{\mathrm{G}} & \equiv \int_{0}^{1} x^{-\beta}\left\{T_{-1}\left(x \mathrm{e}^{-x}\right)\right\}^{\alpha} \mathrm{d} x,
\end{aligned}
$$

where $\alpha>0$ and $\beta$ real for $I_{\mathrm{G}}$, whereas $\alpha>-1$ and $\beta<1$ for $J_{\mathrm{G}}$. Both integrals $I_{\mathrm{G}}$ and $J_{\mathrm{G}}$ are difficult because of the singularities of their integrands at the upper and lower integration limits, respectively. They have been evaluated with an efficient and highly accurate algorithm using the non-standard Gaussian numerical quadratures. Gautschi's programs in matlab, as his open source codes, are available at the web site from Ref. [98].

\subsection{A first-order non-linear differential equation for the Lambert $W(x)$ function}

An alternative interpretation of Eq. (5.15) is possible by multiplying it with $x(1+W)$. The result is the following fundamental first-order ordinary non-linear differential equation satisfied by the Lambert $W$ function:

$$
x[1+W(x)] \frac{\mathrm{d} W(x)}{\mathrm{d} x}=W(x), \quad x \neq 0, \quad x \neq-\frac{1}{\mathrm{e}}
$$

The formulae (5.14)-(5.24) remain valid when real $x$ is replaced by a complex independent variable $z$.

\section{Compositional inverse for general functions}

\subsection{Single-valued inverses}

The expressions (4.33) and (5.2) are the consequence of the existence of the so-called compositional inverses [107]. A given function $F(x)$, defined by its formal power series with zero constant term $\left(a_{0}=0\right)$ : 


$$
F(x)=a_{0}+a_{1} x+a_{2} x^{2}+\cdots=a_{1} x+a_{2} x^{2}+\cdots=\sum_{n=1}^{\infty} a_{n} x^{n}, \quad a_{0}=0,
$$

has a series $G(x)$ for its unique compositional inverse, as denoted by $F^{(-1)}(x) \equiv$ $G(x)$, with the property:

$$
F(G(x))=G(F(x))=x \quad \therefore \quad G(x)=F^{(-1)}(x),
$$

if and only if

$$
a_{1} \neq 0
$$

Since $G(x)$ is the inverse of $F(x)$ via $G(x)=F^{(-1)}(x)$, we can equivalently re-write the relation $G(F(x))=x$ from (6.2) as:

$$
F^{(-1)}(x)(F(x))=x,
$$

or in the same vein

$$
F\left(F^{(-1)}(x)\right)=x
$$

Suppose that a series for $G(x)$ given by:

$$
G(x)=b_{0}+b_{1} x+b_{2} x^{2}+\cdots=b_{1} x+b_{2} x^{2}+\cdots=\sum_{n=1}^{\infty} b_{n} x^{n}, \quad b_{0}=0,
$$

satisfies the relation $F(G(x))=x$ or $G(F(x))=x$ from (6.2). From this assumption, and for $a_{1} \neq 0$, it should follow that the unique inverse $F^{(-1)}(x)$ exists in the form of $G(x)$ via $G(x)=F^{(-1)}(x)$. The coefficients $\left\{b_{n}\right\}(n=1,2,3, \ldots)$ are unknown, but can be determined by substitution of series (6.6) for $G(x)$ into series (6.1) for $F(x)$ and placing the ensuing result into the condition $F(G(x))=x$, thus yielding:

$a_{1}\left(b_{1} x+b_{2} x^{2}+b_{3} x^{3} \cdots\right)+a_{2}\left(b_{1} x+b_{2} x^{2}+\cdots\right)^{2}+a_{3}\left(b_{1} x+\cdots\right)^{3}+\cdots=x$.

When the coefficients of the like powers on both sides of this equation are equated, an infinite system of coupled non-linear equations is deduced:

$$
\left.\begin{array}{c}
a_{1} b_{1}=1 \\
a_{1} b_{2}+a_{2} b_{1}^{2}=0 \\
a_{1} b_{3}+2 a_{2} b_{1} b_{2}+a_{3} b_{1}^{3}=0 \\
\vdots
\end{array}\right\} .
$$


The exact solutions of this system of equations are obtained by progressing successively from the first equation downwards. Thus, the unique solution for $b_{1}$ of the first equation is derived as $b_{1}=1 / a_{1}$ provided that $a_{1} \neq 0$. Inserting this expression for $b_{1}$ into the second equation from the system (6.8) leads to the unique solution $b_{2}=-a_{2} / a_{1}^{3}$ if and only if $a_{1} \neq 0$. Continuing this procedure, it follows:

$$
\left.\begin{array}{c}
b_{1}=\frac{1}{a_{1}} \\
b_{2}=-\frac{a_{2}}{a_{1}^{3}} \\
=2 \frac{a_{2}^{2}}{a_{1}^{5}}-\frac{a_{3}}{a_{1}^{4}} \\
\vdots
\end{array}\right\} \quad \Longleftrightarrow a_{1} \neq 0 .
$$

To show that $G(x)$ is unique, we suppose that in addition to the pair $F(G(x))=x$ and $G(F(x))=x$, there also exists another pair $F(G(x))=x$ and $H(F(x))=$ $x$. However, replacing $x$ by $G(x)$ in $H(F(x))=x$ gives $G(x)=H(F(G(x)))$. Thus, if in the rhs of the latter equation, we insert $F(G(x))=x$, it follows $G(x)=$ $H(F(G(x)))=H(x)$. Therefore, $H(x)=G(x)$ which proves the uniqueness of $G(x)=F^{(-1)}(x)$.

As an example, regarding the Euler tree function $T(x)$, we can find the compositional inverse of some functions made up from $T(x)$, e.g. the rational function $T(x) /[1-T(x)]$. To proceed, we first notice that the function $T(x) /\{1-T(x)\}$ is the composition of $T(x)$ and $x /(1-x)$. On the one hand, as per (3.5), the inverse $\left(x \mathrm{e}^{-x}\right)^{-1}$ of $x \mathrm{e}^{-x}$ is $T(x)$ and, likewise, the inverse $(T(x))^{(-1)}$ of $T(x)$ is $x \mathrm{e}^{-x}$ :

$$
\{T(x)\}^{(-1)}=x \mathrm{e}^{-x} .
$$

Further, from the function $y=x /(1-x)$, we can extract $x$ as $x=y /(1+y)$, meaning that the function $x /(1+x)$ is the inverse function of $x /(1-x)$ :

$$
\left(\frac{x}{1-x}\right)^{(-1)}=\frac{x}{1+x}
$$

Therefore, the compositional inverse of $T(x) /\{1-T(x)\}$ is $\{x /(1+x)\} \exp (-\{x /$ $(1+x)\})$ :

$$
\left\{\frac{T(x)}{1-T(x)}\right\}^{(-1)}=\frac{x}{1+x} \mathrm{e}^{-x /(1+x)} .
$$

\subsection{Multi-valued inverses}

The analysis from 6.1 is valid only if $G(x)$, as the inverse of $F(x)$, is a single-valued function. If $G(x)$ is a multi-valued function, $F(x)$ would not have its inverse. For exam- 
ple, double-valuedness, which gives the same dependent variable $G$ for two different independent variables, i.e. $G\left(x_{1}\right)=G\left(x_{2}\right)$ for $x_{1} \neq x_{2}$, would violate injectiveness (3.7) with the resulting non-existence of $G(x)$, as the inverse of $F(x)$. To rescue this situation and redeem $G(x)$ by transforming it to a true function, which would never have the same value for two different values of the independent variable $x$, we need to impose the condition of single-valuedness onto $G(x)$. This can be achieved by restricting the definition of $G(x)$ to a limited set of values of $x$, rather than including all of them. In the case of a double-valued $G(x)$, there will be only two such restricted ranges of $x$ in each of which $G(x)$ could be single-valued. In this way, both components of $G(x)$, in their respective domains of $x$, would gain the meaning of a genuine function.

The components $G_{k}(x)$ of $G(x)$ represent different branches, where $k$ is the branch counting index, which conventionally takes on any integer value $(k=$ $0, \pm 1, \pm 2, \ldots)$. Overall, a multi-valued function $G(x)$ can be regularized by introduction of a sequence (possibly infinite) of single-valued branches $G_{k}(x)(k=$ $0, \pm 1, \pm 2, \ldots)$. In other words, the function $F(x)$ will not have the unique inverse, but nevertheless could possess many well-defined inverses as different branches. However, such circumstances change the meaning of the compositional inverses in (6.2), such that e.g. the relation $G_{k}(F(x))=x$ could still be valid, but only for certain values of $x$, whereas for some other $x$, we could have $G_{k}(F(x)) \neq x$. This will be illustrated in Sect. 7 with the examples of $F(x)$ chosen to be the functions $x \mathrm{e}^{x}$ and $x \mathrm{e}^{-x}$ from (3.1) and (3.2), the multi-valued inverses of which are given by the Lambert $W(x)$ and Euler $T(x)$ functions (3.4) and (3.5), respectively.

\section{Compositional inverses $T_{k}\left(x e^{-x}\right)$ and $W_{k}\left(x e^{x}\right)$ for $k=0$ and $k=-1$}

\subsection{A key link between a branch choice and the independent variable domain}

In order to specify the general analysis from Sect. 6.2, we shall now apply the notion of a compositional inverse to the Euler $T$ and Lambert $W$ functions. Since the Euler function $T(y)$ is the solution $x$ of the transcendental equation (4.32), we can replace $y$ by $x \mathrm{e}^{-x}$ in $T(y)=x$ and write:

$$
T\left(x \mathrm{e}^{-x}\right)=x .
$$

Similarly, because the Lambert function $W(y)$ is the solution $x$ of the transcendental equation (5.1), substitution of $y$ by $x \mathrm{e}^{x}$ in $W(y)=x$ would yield:

$$
W\left(x \mathrm{e}^{x}\right)=x
$$

This brief derivation of the relations (7.1) and (7.2) is formally correct. However, since $T$ and $W$ are multi-valued functions, a proper validation of (7.1) and (7.2) can be secured only after a specific branch has been selected. More precisely, a mere branch selection would be able to confirm or disprove (7.1) and (7.2), as we shall now demonstrate. Moreover, a branch choice would automatically determine the $x$ intervals for which the relations (7.1) and (7.2) are correct or wrong. As such, caution 
has to be exercised as to a tempting, but otherwise incorrect conclusion that e.g. the two most important compositional inverses $T_{k}\left(x \mathrm{e}^{-x}\right)=x$ and $W_{k}\left(x \mathrm{e}^{x}\right)=x$ from (7.1) and (7.2) could be valid without the need to specify the domains for $x$. Recall that the general compositional inverse $G_{k}(F(x))=x$ from (6.2) is not valid either for a multiple-valued inverse $G_{k}(x)=F^{(-1)}(x)$ for every $x$.

Similar validity limitations also apply to functions $x \mathrm{e}^{x}$ and $x \mathrm{e}^{-x}$ from (3.1) and (3.2), because they possess infinitely many inverses $W_{k}(x)$ and $T_{k}(x)$ for different branches $k(k=0, \pm 1, \pm 2, \ldots)$. Given that most applications are concerned with real-valued Euler $T(x)$ and Lambert $W(x)$ functions for real $x$, we will specify the validity criteria that are applicable to the two branches $k=0$ and $k=-1$ for both $T_{k}(x)$ and $W_{k}(x)$. In order to determine for which $x$ the compositional inverse (7.1) is valid, we apply the mapping $T$ on both sides of equation $y=x \mathrm{e}^{-x}$ from (4.32) and use the definitions in (5.11) to identify $T_{0}$ and $T_{-1}$, so that:

$$
T\left(x \mathrm{e}^{-x}\right)=T(y)= \begin{cases}T_{0}(y), & T(y) \leq 1 \\ T_{-1}(y), & T(y) \geq 1 .\end{cases}
$$

Therefore, the relationship $T\left(x \mathrm{e}^{-x}\right)=T(y)$ from (7.3) would become $T_{0}\left(x \mathrm{e}^{-x}\right)=x$, only if $x$, taken here to be $T_{0}(y)$, is less than or equal to unity, $x=T_{0}(y) \leq 1$. In other words, if $x$ is going to be $T_{0}(y)$, in order to be able to transform $T_{0}\left(x \mathrm{e}^{-x}\right)=T_{0}(y)$ into $T_{0}\left(x \mathrm{e}^{-x}\right)=x$, we must have $x \leq 1$. This occurs because, by the definition, we have that $T_{0}(y) \leq 1$, as per (5.11). In contrast to this, however, for the complementary interval $x>1$, we would have $T_{0}\left(x \mathrm{e}^{-x}\right)=\widetilde{T}_{0} \neq x$, where $\widetilde{T}_{0}$ is the result of an explicit computation of $T_{0}(X)$ from the appropriate representation of $T_{0}(X)$, as dictated by the given $X$, which itself is determined by the value of $x$ through the relation $X=x \mathrm{e}^{-x}$. Hence, for the branch $T_{0}$, the compositional inverse (7.1) is not valid in a general case with no restriction imposed on $x$. Rather it is applicable only to $x \leq 1$, whereas for the complementary domain $x>1$, we have $T_{0}\left(x \mathrm{e}^{-x}\right) \neq x$ :

$$
T_{0}\left(x \mathrm{e}^{-x}\right)=\left\{\begin{array}{ll}
x, & x \leq 1 \\
\widetilde{T}_{0}, & x>1,
\end{array} \quad \widetilde{T}_{0} \neq x\right.
$$

In other words, while the relation $T_{0}\left(x \mathrm{e}^{-x}\right)=x$ holds true for $x \leq 1$, it must be replaced by $T_{0}\left(x \mathrm{e}^{-x}\right)=\widetilde{T}_{0}$ for $x>1$, where $\widetilde{T}_{0} \neq x$. Similarly, the alternative path $T_{-1}\left(x \mathrm{e}^{-x}\right)=T_{-1}(y)$ from (7.3) would simplify to $T_{-1}\left(x \mathrm{e}^{-x}\right)=x$ only if $x=T_{-1}(y) \geq 1$, for otherwise $T_{-1}\left(x \mathrm{e}^{-x}\right)=\widetilde{T}_{-1} \neq x$ if $x=T_{-1}(y)<1$. The meaning of $\widetilde{T}_{-1}$ is analogous to $\widetilde{T}_{0}$. Here, in order to have $T_{-1}\left(x \mathrm{e}^{-x}\right)=x$, instead of $T_{-1}\left(x \mathrm{e}^{-x}\right)=T_{-1}(y)$, which amounts to the condition $x=T_{-1}(y)$, we must impose the restriction $x \geq 1$ since, by definition, $T_{-1}(y) \geq 1$ as seen in (5.11). Thus, we have:

$$
T_{-1}\left(x \mathrm{e}^{-x}\right)=\left\{\begin{array}{ll}
x, & x \geq 1 \\
\widetilde{T}_{-1}, & x<1,
\end{array} \quad \widetilde{T}_{-1} \neq x\right.
$$


This can equivalently be written as $T_{0}\left(x \mathrm{e}^{-x}\right)=x$ only for $x \in[0,1]$ and $T_{-1}\left(x \mathrm{e}^{-x}\right)=$ $x$ only for $x \in[1, \infty)$, in accordance with Ref. [83,97].

A reasoning, entirely analogous to that for $T(x)$, can also be applied to $W(x)$ to establish the restrictions on (7.2) for the corresponding compositional inverses $W_{0}(x)$ and $W_{-1}(x)$ with the help of the defining relation (5.9) of these two latter branches. Alternatively, and more directly, we can use the relationship $W(x)=-T(-x)$ from (3.6) to immediately write the compositional inverses for $W_{0}(x)$ and $W_{-1}(x)$ based upon (7.4) and (7.5), respectively. Therefore, if e.g. in $T_{0}\left(x \mathrm{e}^{-x}\right)=x$, valid for $x \leq 1$, as per (7.4), we replace $x$ by $-x$ and use (3.6), we would obtain the relation $W_{0}\left(x \mathrm{e}^{x}\right)=$ $x$, valid for $-x \leq 1$ (or equivalently, for $x \geq-1$ ). Applying a similar rationale to the branches $T_{-1}$ and $W_{-1}$, we can deduce that the equation $W_{-1}\left(x \mathrm{e}^{x}\right)=x$ is valid only for $x \leq-1$. However, if these conditions for $x$ are not fulfilled, then $W_{k}\left(x \mathrm{e}^{x}\right)$ must be computed explicitly from the pertinent formulae for the Lambert functions with the outcome $\widetilde{W}_{k}$ which is different from $x$. In this way, we can arrive at the expressions for the compositional inverses $W_{0}\left(x \mathrm{e}^{x}\right)$ and $W_{-1}\left(x \mathrm{e}^{x}\right)$ in the following forms:

$$
W_{0}\left(x \mathrm{e}^{x}\right)=\left\{\begin{array}{ll}
x, & x \geq-1 \\
\widetilde{W}_{0}, & x<-1,
\end{array} \quad \widetilde{W}_{0} \neq x,\right.
$$

and

$$
W_{-1}\left(x \mathrm{e}^{x}\right)=\left\{\begin{array}{ll}
x, & x \leq-1 \\
\widetilde{W}_{-1}, & x>-1,
\end{array} \quad \widetilde{W}_{-1} \neq x\right.
$$

Recall that quantities $\widetilde{T}_{k} \neq x$ and $\widetilde{W}_{k} \neq x$, that were first introduced in (5.12) and (5.13), as the abbreviated notations for the values of functions $T_{k}\left(x \mathrm{e}^{-x}\right)$ and $W_{k}\left(x \mathrm{e}^{x}\right)$, must be computed from the appropriate formulae of $T_{k}\left(x_{\mathrm{m}}\right)$ and $W_{k}\left(x_{\mathrm{p}}\right)$ taken at arguments $x_{\mathrm{m}}=x \mathrm{e}^{-x}$ and $x_{\mathrm{p}}=x \mathrm{e}^{x}$, respectively.

Overall, from the onset of this sub-section we correctly (albeit formally) derived (7.1) and (7.2), as the compositional inverses. We say 'formally' because, when we started addressing this matter, the mentioned derivation has not specified the possible ranges for $x$. Yet, such an establishment of the results for $T\left(x \mathrm{e}^{-x}\right)$ and $W\left(x \mathrm{e}^{x}\right)$ via (7.1) and (7.2), respectively, is not universally valid for every $x$. The reason is rooted in the multi-valuedness of $T$ and $W$ through the existence of infinitely many branches $T_{k}(x)$ and $W_{k}(x)(k=0, \pm, \pm 2, \ldots)$.

However, a simplification occurs for real $x$ in $T(x)$ and $W(x)$. In this case, a mere choice of the only two possible values for the branch counter $k$ ( $k=0$ and $k=-1$ ) suffices to determine the interval of $x$ where the compositional inverses (7.1) and (7.2) exist. Hence, e.g. the choice $k=0$ automatically determines that the relation $T_{0}\left(x \mathrm{e}^{-x}\right)=x$ is valid for $x \leq 1$ because of the existence of the inequality $T_{0}\left(x \mathrm{e}^{-x}\right) \leq 1$, or more generally, $T_{0}(X) \leq 1$ for any $X$ for which $T_{0}(X)$ is defined. The relation $T_{0}\left(x \mathrm{e}^{-x}\right) \leq 1$ follows from the definition $T(x)=T_{0}(x)$ for $T(x) \leq 1$, as per (5.11). In the same vein, selection of the branch counting index $k=-1$ yields $T_{-1}\left(x \mathrm{e}^{-x}\right)=x$ for $x>1$ due to $T_{-1}\left(x \mathrm{e}^{-x}\right)>1$, or $T_{-1}(X)>1$ for any $X$ from the 
domain of definition $T_{-1}(X)$. The inequality $T_{-1}\left(x \mathrm{e}^{-x}\right)>1$ stems from the definition $T(x)=T_{-1}(x)$ for $T(x)>1$, which is in (5.11).

Whenever for the given branch index $k$, the mentioned restrictions on $x$ are not fulfilled, the compositional inverse does not exist and instead the relation $T_{k}\left(x \mathrm{e}^{-x}\right)=$ $\widetilde{T}_{k}$ is obtained with $\widetilde{T}_{k} \neq x(k=0,-1)$. This is the content of (7.4) and (7.5). These considerations would automatically yield the corresponding expressions (7.6) and (7.7) for the Lambert function $W_{k}\left(x \mathrm{e}^{x}\right)$ with $k=0$ and $k=-1$ when replacing $x$ by $-x$ and using the relation $W(X)=-T(-X)$ from (3.6).

\subsection{Explicit proof of the compositional inverse for the Lambert function}

Here, we will show how formally the sum (4.14) and (5.4) can be carried out algebraically for a special case $y=x \mathrm{e}^{-x}$ and $y=x \mathrm{e}^{x}$, respectively:

$$
\begin{aligned}
T\left(x \mathrm{e}^{-x}\right) & =\sum_{n=1}^{\infty} \frac{n^{n-1}}{n !}\left(x \mathrm{e}^{-x}\right)^{n}, \\
W\left(x \mathrm{e}^{x}\right) & =\sum_{n=1}^{\infty} \frac{(-n)^{n-1}}{n !}\left(x \mathrm{e}^{x}\right)^{n} .
\end{aligned}
$$

To this end, we first insert the MacLaurin series for the exponential in the function $T\left(x \mathrm{e}^{-x}\right)$, which for brevity, we denote by $x^{\prime}$ :

$$
x^{\prime} \equiv T\left(x \mathrm{e}^{-x}\right)
$$

so that

$$
\begin{aligned}
x & =\sum_{n=1}^{\infty} \frac{n^{n-1}}{n !}\left(x \mathrm{e}^{-x}\right)^{n} \\
& =\left\{\sum_{n=1}^{\infty} \frac{n^{n-1}}{n !} x^{n}\right\}\left\{\sum_{\ell=0}^{\infty} \frac{(-n x)^{\ell}}{\ell !}\right\} \\
& =\sum_{n=1}^{\infty} \sum_{\ell=0}^{\infty}(-1)^{\ell} \frac{x^{n+\ell}}{n ! \ell !} n^{n+\ell-1} \\
& =\sum_{k=1}^{\infty} \frac{x^{k}}{k !} \sum_{m=1}^{k}(-1)^{k-m} \frac{k !}{m !(k-m) !} m^{k-1} \\
& =\sum_{k=1}^{\infty} \frac{x^{k}}{k !}\left\{\sum_{m=1}^{k}(-1)^{k-m}\left(\begin{array}{c}
k \\
m
\end{array}\right) m^{k-1}\right\} .
\end{aligned}
$$


Thus, we can express $x^{\prime}$ as:

$$
x^{\prime}=\sum_{k=1}^{\infty} \frac{x^{k}}{k !} I_{k}
$$

where

$$
I_{k}=\sum_{m=1}^{k}(-1)^{k-m}\left(\begin{array}{c}
k \\
m
\end{array}\right) m^{k-1}, \quad\left(\begin{array}{c}
k \\
m
\end{array}\right)=\frac{k !}{m !(k-m) !} .
$$

The sum over $m$ in (7.12) can be extended to encompass $m=0$ by adding and subtracting the term $\left\{(-1)^{k-m} m^{k-1}\right\}_{m=0}$, which has the following values:

$$
\left\{(-1)^{k-m} m^{k-1}\right\}_{m=0}=(-1)^{k} 0^{k-1}= \begin{cases}-1, & k=1 \\ 0, & k \geq 2\end{cases}
$$

where $0^{n}=0$ for $n>0$ ( $n$ non-negative integer) and $0^{0} \equiv 1$. Thus, it follows:

$$
\begin{aligned}
I_{k} & =\sum_{m=0}^{k}\left\{(-1)^{k-m}\left(\begin{array}{c}
k \\
m
\end{array}\right) m^{k-1}-(-1)^{k} 0^{k-1}\right\} \\
& =\sum_{m=0}^{k}\left\{(-1)^{k-m}\left(\begin{array}{c}
k \\
m
\end{array}\right) m^{k-1}+\delta_{k, 1}\right\},
\end{aligned}
$$

where the term $(-1)^{k} 0^{k-1}$ from (7.13) is equivalently written as the Kronecker $\delta$ symbol, $(-1)^{k} 0^{k-1}=-\delta_{k, 1}$ with:

$$
\delta_{k, k^{\prime}}= \begin{cases}1, & k=k^{\prime} \\ 0, & k \neq k^{\prime}\end{cases}
$$

The Kronecker term in (7.14) is independent of the summation index $m$, so that:

$$
I_{k}=\delta_{k, 1}+J_{k}
$$

where

$$
J_{k}=\sum_{m=0}^{k}(-1)^{k-m}\left(\begin{array}{c}
k \\
m
\end{array}\right) m^{k-1}
$$


For $k=1$, the explicit calculation shows that:

$$
\begin{gathered}
J_{1}=\sum_{m=0}^{1}(-1)^{1-m}\left(\begin{array}{c}
1 \\
m
\end{array}\right) m^{0}=-\left(\begin{array}{l}
1 \\
0
\end{array}\right)+\left(\begin{array}{l}
1 \\
1
\end{array}\right)=-1+1=0, \\
\therefore \quad J_{1}=0 .
\end{gathered}
$$

We insert (7.16) into (7.11) to write:

$$
x^{\prime}=x+x^{\prime \prime}
$$

with

$$
x^{\prime \prime}=\sum_{k=2}^{\infty} \frac{x^{k}}{k !} J_{k},
$$

where the result (7.18) is used. To analyze the sum over $m$ in $J_{k}$ from (7.17) for any $k$, we introduce the first difference operator $\widehat{\Delta}$ for an arbitrary function $f(n)$ by the standard definition:

$$
\begin{aligned}
\widehat{\Delta} f(n) & =f(n+1)-f(n) \\
& =\widehat{\mathrm{E}} f(n)-\widehat{\mathrm{E}} f(n-1),
\end{aligned}
$$

where $\widehat{\mathrm{E}}$ is the shift operator [107]

$$
\widehat{\mathrm{E}} f(n)=f(n+1) \text {. }
$$

As is clear from (7.21), the operators $\widehat{\Delta}$ and $\widehat{E}$ are connected by:

$$
\widehat{\Delta}=\widehat{\mathrm{E}}-\widehat{1}
$$

where $\widehat{1}$ is the unity operator, $\widehat{1} f(n)=f(n)$. The $k$ th power of the operator $\widehat{\mathrm{E}}$ is very simple, since the repeated use of the definition (7.22) produces merely the scaling from $n$ to $n+k$ in $f(n)$ :

$$
\widehat{\mathrm{E}}^{k} f(n)=f(n+k) .
$$

By contrast, the $k$ th power of the operator $\widehat{\Delta}$ is more complicated. Nevertheless, the explicit formula for $\widehat{\Delta}^{k} f(n)$ can be derived as follows. If the application of the $\widehat{\Delta}$ operator on $f(n)$ is performed $k$ times, the $k$ th difference operator $\widehat{\Delta}^{k}$ would become available by the iteration:

$$
\widehat{\Delta}^{k} f(n)=\widehat{\Delta}\left\{\widehat{\Delta}^{k-1} f(n)\right\}=\widehat{\Delta}^{k-1}\{\widehat{\Delta} f(n)\},
$$


or equivalently, by way of (7.23)

$$
\widehat{\Delta}^{k} f(n)=(\widehat{\mathrm{E}}-\widehat{1})^{k} f(n)
$$

We see that the action of $\widehat{\Delta}^{k}$ on $f(n)$ is not as direct as in the case of $\widehat{\mathrm{E}}^{k}$. The reason is in the fact that the rhs of (7.26) contains the binomial operator $(\widehat{\mathrm{E}}-\widehat{1})^{k}$, instead of having only $\widehat{\mathrm{E}}^{k}$ from (7.24). The meaning of the $k$ th power of the operator $\widehat{\mathrm{E}}-\widehat{1}$ is provided by the operator Leibniz binomial formula, which has the same form as its scalar counterpart:

$$
(a+b)^{k}=\sum_{m=0}^{k}\left(\begin{array}{c}
k \\
m
\end{array}\right) a^{m} b^{k-m}
$$

so that

$$
\begin{aligned}
\widehat{\Delta}^{k} f(n) & =(\widehat{\mathrm{E}}-\widehat{1})^{k} f(n) \\
& =\sum_{m=0}^{k}\left(\begin{array}{c}
k \\
m
\end{array}\right)(-1)^{k-m} \widehat{\mathrm{E}}^{m} f(n),
\end{aligned}
$$

where we used the relations $\widehat{\mathrm{E}}^{m}\left(-\widehat{1}^{k-m} f(n)=\widehat{\mathrm{E}}^{m}(-1)^{k-m} f(n)=(-1)^{k-m} \widehat{\mathrm{E}} f(n)\right.$. Here, the term $\widehat{\mathrm{E}}^{m} f(n)$ is recognized as $f(n+m)$ according to (7.24), so that:

$$
\widehat{\Delta}^{k} f(n)=\sum_{m=0}^{k}\left(\begin{array}{c}
k \\
m
\end{array}\right)(-1)^{k-m} f(n+m) .
$$

This compact formula for $\widehat{\Delta}^{k} f(n)$ is explicit, since instead of operators, the rhs of (7.28) involves only a linear combination of $k$ scalar function values $\{f(n+m)\}(0 \leq$ $m \leq k$ ) for a fixed $n$. In particular, for $n=0$, the $k$ difference $\widehat{\Delta}^{k} f(0)$ is reduced to:

$$
\widehat{\Delta}^{k} f(0)=\sum_{m=0}^{k}\left(\begin{array}{l}
k \\
m
\end{array}\right)(-1)^{k-m} f(m) .
$$

Choosing $f(m)$ in (7.29) to be a power function of $m$ :

$$
f(m)=m^{k-1}
$$

it follows

$$
\widehat{\Delta}^{k} 0^{k-1}=\sum_{m=0}^{k}\left(\begin{array}{c}
k \\
m
\end{array}\right)(-1)^{k-m} m^{k-1} .
$$


The rhs of Eq. (7.31) coincides with the sum in $J_{k}$ from (7.17) and this implies:

$$
J_{k}=\widehat{\Delta}^{k} 0^{k-1} .
$$

In Eq. (7.20) for $x^{\prime \prime}$, we need $J_{k}$ for $k \geq 2$, in which case the term $0^{k-1}$ is zero. This implies $\widehat{\Delta}^{k} 0^{k-1}=\widehat{\Delta}^{k} 0=0(k \geq 2)$, and consequently the rhs in Eq. (7.32) is also equal to zero, so that:

$$
J_{k} \equiv \sum_{m=0}^{k}(-1)^{k-m}\left(\begin{array}{c}
k \\
m
\end{array}\right) m^{k-1}=0, \quad \forall k \geq 2
$$

We have found earlier in (7.18) that $J_{1}=0$, and this extends (7.33) to $J_{k}=0(\forall k \geq 1)$, although the case with $k=1$ is not needed in $x^{\prime \prime}$ from (7.20). Therefore, by substituting the result (7.33) for $J_{k}$ in the expression for $x^{\prime \prime}$, the whole sum over $k$ from (7.20) collapses to zero, thus yielding:

$$
x^{\prime \prime}=0
$$

which reduces (7.19) to

$$
x^{\prime}=x
$$

Quantity $x^{\prime}$, as the lhs of (7.35), is equal to $T\left(x \mathrm{e}^{-x}\right)$ by reference to (7.10), so that:

$$
T\left(x \mathrm{e}^{-x}\right)=x .
$$

With this at hand, we have the sought algebraically calculated result for the sum rule (7.8) as:

$$
T\left(x \mathrm{e}^{-x}\right)=\sum_{n=1}^{\infty} \frac{n^{n-1}}{n !}\left(x \mathrm{e}^{-x}\right)^{n}=x .
$$

Likewise, a calculation along these lines can algebraically prove the sum rule (7.9). This is, however, unnecessary since we can use the relation $-T(-x)=W(x)$ from (3.6) to immediately deduce the final results from (7.36) and (7.37) as follows:

$$
W\left(x \mathrm{e}^{x}\right)=x,
$$

and

$$
W\left(x \mathrm{e}^{x}\right)=\sum_{n=1}^{\infty} \frac{(-n)^{n-1}}{n !}\left(x \mathrm{e}^{x}\right)^{n}=x .
$$


In the outlined analysis, the algebraically derived sum rules (7.37) and (7.39) are, in fact, the power series representation of the compositional inverses $T\left(x \mathrm{e}^{-x}\right)=x$ and $W\left(x \mathrm{e}^{x}\right)=x$ from (7.36) and (7.38), respectively [107].

Nevertheless, here too, caution should be exercised as to the general validity of the derivation in this sub-section, similarly to the corresponding remarks made in Sect. 7.1. Namely, although considerably longer, this derivation is still only formal. The reason is in the occurrence that the compositional inverses (7.36) and (7.38), or equivalently, the sum rules (7.37) and (7.39), are established for any branch of the multi-valued Euler and Lambert functions and, moreover, with no regard whatsoever to the limitations on the ranges for $x$. However, as per Sect. 7.1, even for a given, fixed branch of $T_{k}$ or $W_{k}$, the obtained result $x$ for the compositional inverses (7.36) or (7.38) is not valid for any $x$. Thus, by reference to Sect. 7.1, the validity of (7.36) and (7.38) for e.g. $k=\{0,1\}$ is specified strictly through $\{(7.4),(7.5)\}$ and $\{(7.6),(7.7)\}$, respectively.

\section{Asymptotic behavior of $W(x)$ and $T(x)$ at small and large $x$}

Here, whenever convenient for connecting with Refs. [107] and [110], we will switch from the Lambert $W$ to the Euler $T$ function, which is always permissible on the account of their inter-relationship (3.6), i.e. $-W(-y)=T(y)$. Thus, we shall begin with $W$, then continue with $T$, and finally return to $W$. In particular, the asymptotic behaviors of e.g. $W(x)$ for small and large $x$ can be obtained from the defining expressions (5.2) and (5.3), respectively, after rewriting them as follows:

$$
\begin{aligned}
& W(x)=\frac{x}{\mathrm{e}^{W(x)}}=\frac{x}{\exp (W(x))}, \\
& W(x)=\ln x-\ln W(x)=\ln \frac{x}{W(x)} .
\end{aligned}
$$

These two transcendental equations can be solved for $W(x)$ by means of iterations through self-unrolling. This can be done by repeatedly inserting $x / \mathrm{e}^{W(x)}$ in the argument $W(x)$ of the exponential in the denominator of the rhs of Eq. (8.1). The result is the following representation of $W(x)$ in terms of the infinitely iterated exponential function via $W=x /\{\exp (W)\}=x /\{\exp (x /[\exp (W)])\}$, i.e.:

$$
W(x)=\frac{x}{\exp \left(\frac{x}{\exp (W(x))}\right)}=\cdots=\frac{x}{\exp \left(\frac{x}{\exp \left(\frac{x}{\ddots}\right)}\right)}
$$


or equivalently

$$
W(x)=x \mathrm{e}^{-x \mathrm{e}^{-W(x)}}=\cdots=x \mathrm{e}^{-x \mathrm{e}^{-x \mathrm{e}^{-x} \cdots}} \quad \text { (Continued exponential) } .
$$

If here we reverse the sign of $x$ (i.e. $x \rightarrow-x$ ), and afterward use the relation $-W(-x)=T(x)$, then the expression (8.4) would become:

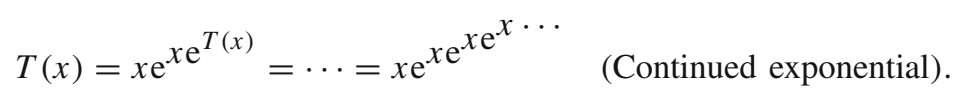

The meaning of the expression (8.5) can be understood by introducing an auxiliary function $\tilde{t}_{m}(x)$ by the recursion [107]:

$$
\tilde{t}_{m+1}(x)=x \mathrm{e}^{\tilde{t}_{m}(x)}, \quad m \geq 0, \quad \tilde{t}_{0}(x)=x .
$$

Then, for a fixed $x$, function $T(x)$ from (8.5) is defined as the following limit of the sequence $\left\{\tilde{t}_{m}(x)\right\}(m=0,1,2,3, \ldots)$ :

$$
\lim _{m \rightarrow \infty} \tilde{t}_{m}(x)=T(x),
$$

whenever the limit exists in a formal sense. This is recognized as the root-finding Newton iteration process. Here, $x$ from the initialization $\tilde{t}_{0}=x$ is taken to be a known trial value $x_{\text {trial }}$, which enables the iteration to start from $\tilde{t}_{0}=x_{\text {trial }}$. For instance, the approximate root of Eq. (4.32), re-written as:

$$
x=y \mathrm{e}^{x},
$$

can be found by a straightforward application of the Newton algorithm

$$
x_{m+1}=y \mathrm{e}^{x_{m}}, \quad m \geq 0, \quad x_{0}=y .
$$

Here, the trial value $x_{\text {trial }}$ for the zeroth iterate $x_{0}$ is chosen as the solution of Eq. (8.8) with its rhs taken at $x=0$ :

$$
x_{0}=x_{\text {trial }}=\left\{y \mathrm{e}^{x}\right\}_{x=0} \quad \therefore \quad x_{0}=x_{\text {trial }}=y .
$$

The 1st iterate $x_{1}$ is obtained from the recursion (8.9) by inserting $x_{0}$ to give $x_{1}=$ $y \exp \left(x_{0}\right)=y \exp (y)$. When the approximation $x_{1}$ is inserted into $y \mathrm{e}^{x_{1}}$ from (8.9), the 2 nd iterate follows as $x_{2}=y \exp (y \exp (y))$. An analogous procedure for $n=3$ gives the 3rd Newton iterate via:

$$
x_{3}=y \exp (y \exp (y \exp (y)))=y \mathrm{e}^{y \mathrm{e}^{y \mathrm{e}^{y}}} .
$$


Continuing iteratively in this way some $n$ times would give the $n$th iterate $x_{n}$ which is the $n$th Newton approximation to the exact root $x_{\text {exact }}=T(y)$ of (8.8). The root estimate as the $n$th Newton iterate can be written by the symbolic expression $(y \exp )^{\{n\}} y:$

$$
x \approx x_{n}=(y \exp )^{\{n\}} y=\underbrace{y \exp (y \exp (y \exp (\cdots)))}_{n \text { terms }} y,
$$

where $(y \exp )^{\{n\}}$ is an operator which abbreviates the $n$ nested exponential operators [110]:

$$
(y \exp )^{\{n\}}=\underbrace{y \exp (y \exp (y \exp (\cdots)))}_{n \text { terms }} .
$$

Here, the curly brackets are used around $n$ in the superscript to avoid confusion with a power function. Thus, for e.g. $n=3$, it follows that Eq. (8.10) simplifies as:

$$
\begin{aligned}
x_{3}=(y \exp )^{\{3\}} y & =\underbrace{y \exp (y \exp (y \exp ))}_{3 \text { terms }} y \\
& =y \exp (y \exp (y \exp (y))) \\
& =y \mathrm{e}^{y \mathrm{e}^{y \mathrm{e}^{y}},}
\end{aligned}
$$

and this agrees with (8.11). Of course, a specific fixed number for the initial value $x_{0}$ is needed from the outset of an actual numerical generation of the Newton iterates $x_{n}(n=0,1,3, \ldots)$, as the successive approximations to the exact solution $x$ of Eq. (8.8) given by the Euler $T(y)$ function.

However, in an analytical calculation aiming at deriving an algebraic explicit solution $x$ of Eq. (8.8) in a closed form, no such specification is needed for $x_{0}$ at the very beginning of the iteration process. To this end, it suffices to choose $x_{0}$ as an unspecified value, namely the unknown $x$ which is the solution of the problem:

$$
x_{m+1}=y \mathrm{e}^{x_{m}}, \quad m \geq 0, \quad x_{0}=x .
$$

Then repeating the outlined Newton procedure, but this time with $x_{0}=x$, as per (8.15), yields the $n$th iterate $x_{n}$ as the result of application of the operator (8.13) to $x$ :

$$
x \approx x_{n}=(y \exp )^{\{n\}} x=\underbrace{y \exp (y \exp (y \exp (\cdots)))}_{n \text { terms }} x .
$$

In theory, the value $n=\infty$ is permitted, in which case $x_{\infty}$ would be the result of infinitely many iterations:

$$
x_{\infty}=(y \exp )^{\{\infty\}} x=\underbrace{y \exp (y \exp (y \exp (\cdots)))}_{n=\infty: \text { infinitely many terms }} x .
$$


Expression (8.16) is the analytical solution $x$ of Eq. (8.8) in $n$ steps of the Newton iteration (8.15). For an infinitely large $n$ the solution $x_{n}$ becomes $x_{\infty}$, where $x_{\infty}$ is from (8.17). However, the result (8.16) itself is an implicit transcendental expression, since the unknown $x$ is a part of the solution $x=(y \exp )^{\{n\}} x$. Moreover, the solution (8.16) is much more complicated than the original implicit transcendental equation (8.8), since we now have an iterated exponential rather than just one such initial exponential.

Nevertheless, this obstacle can be circumvented by noting that $x$ in the solution $(y \exp )^{\{n\}} x$ can be traced back to the unspecified initialization $x_{0}=x$ in the Newton iteration (8.16). Therefore, setting $x=x_{0}$ in the implicit function $(y \exp )^{\{n\}} x$ will transform the $n$th iterate $x_{n}$ from (8.16) into the following explicit approximate solution of Eq. (8.8):

$$
x_{n}=(y \exp )^{\{n\}} x_{0}=\underbrace{y \exp (y \exp (y \exp (\cdots)))}_{n \text { terms }} x_{0},
$$

and similarly for infinitely many iterations

$$
x_{\infty}=(y \exp )^{\{\infty\}} x_{0}=\underbrace{y \exp (y \exp (y \exp (\cdots)))}_{n=\infty: \text { infinitely many terms }} x_{0},
$$

where $x_{0}$ is a free parameter. Now, at the end of the derivation, we can specify $x_{0}$ by the prescription (8.10), via $x_{0}=y$, so that:

$$
x_{\infty}=(y \exp )^{\{\infty\}} x_{0}=\underbrace{y \exp (y \exp (y \exp (\cdots)))}_{n=\infty \text { infinitely many terms }} y .
$$

By reference to (8.5), the rhs of Eq. (8.20) is the Euler $T(y)$ function as the exact solution of the transcendental equation (8.8), which we set to solve for the unknown $x$, so that:

$$
x_{\infty}=T(y)
$$

Once the solution $x_{\infty}$ was identified with one of the representations of $T(y)$, namely the continued exponentials (8.5), it is permissible to cast $x_{\infty}$ into any of the other existing forms of $T(y)$. In other words, $x_{\infty}$ is $T(y)$ irrespective of the selected representation of $T(y)$. Customarily, the Newton root-finding iterations are used as a numerical algorithm. The above derivation shows that the same algorithm can also be employed for obtaining the exact analytical solutions to the roots of a class of implicitly defined functions.

As an alternative to the outlined procedure, we can also substitute repeatedly $\ln x-$ $\ln W(x)$ in the argument $W(x)$ of the second logarithm on the rhs of Eq. (8.2). This expresses $W(x)$ as an infinitely iterated logarithm function: 


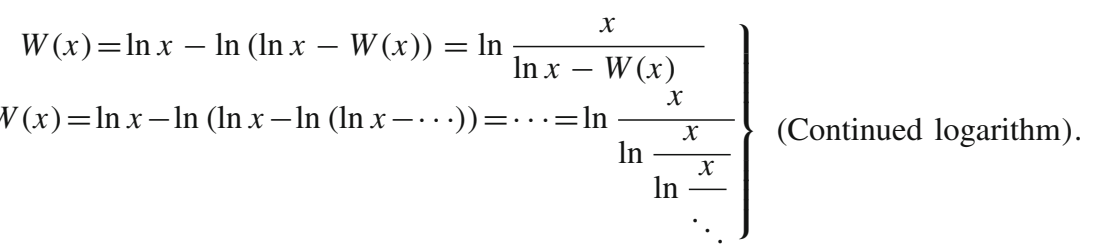

The iterative forms (8.3) and (8.22) are of a type of a continued fraction (CF) [111]. For the reason of economizing space, it is customary to use a shortened symbolic notation for a CF-type expression. In such a notation, the staircase iterated ratios from (8.3) and (8.22) can succinctly be written as:

$$
W(x)=x / \exp (x / \exp (x / \exp (x / \cdots)))
$$

and

$$
W(x)=\ln (x / \ln (x / \ln (x / \cdots))),
$$

respectively. In the above formulae $x$ is real-valued. If $x$ is replaced by a complex variable $z$, the definition (5.2) continues to be of the same form, whereas (5.3) is modified as [80]:

$$
\left.\begin{array}{c}
W_{k}(z) \mathrm{e}^{W_{k}(z)}=z, \quad \ln W_{k}(z)+W_{k}(z)=\operatorname{Ln} z \\
\operatorname{Ln} z=\ln z+2 i k \pi, \quad i=+\sqrt{-1}
\end{array}\right\} \quad(k=0, \pm 1, \pm 2, \ldots)
$$

Thus, whenever the symbol Ln is used instead of $\ln$, the branch is left unspecified. The standard principal branch of the natural logarithmic function $\ln$ is the one which is cut along the negative real semi-axis (i.e. from 0 to $-\infty$ ).

This latter convention also applies to the square root function $u$ from (5.8) whenever $x$ is negative or complex-valued. The relation $W(x)=\ln (x / W(x))$ for real $x$ from (8.2) is not preserved for complex $z$ unless $k=0$ (principal branch), because of (8.25) which implies:

$$
W_{k}(z)=\ln \frac{z}{W_{k}(z)}+2 i k \pi \quad(k=0, \pm 1, \pm 2, \cdots) .
$$

The iterative CF-type forms (8.3) and (8.22) of the Lambert function are suitable for extraction of the corresponding asymptotic behaviors at small and large values of real variable $x$, respectively:

$$
\left.\begin{array}{rl}
W(x) \underset{x \rightarrow 0}{\underset{\rightarrow}{\approx}} \frac{x}{1+x} \\
\underset{x \rightarrow 0}{\approx} x-x^{2}
\end{array}\right\}
$$


and

$$
\left.\begin{array}{rl}
W(x)_{x} \underset{\rightarrow \infty}{\approx} \ln x-\ln (\ln x) \\
\underset{x \rightarrow \infty}{\approx} \ln \frac{x}{\ln x}
\end{array}\right\} .
$$

We see that the leading terms of the asymptotes of $W(x)$ from (8.27) and (8.28) for small and large $x$ are $x$ and $\ln x$, respectively. Hence, it is through its small- and large- $x$ asymptotes that the Lambert function $W(x)$ captures the linear-logarithmic feature of the lhs of the transcendental, implicit equation:

$$
y+\ln y=\ln x
$$

whose solution $y$ is $W(x)$, i.e. $y=W(x)$, according to the definition (5.3). In fact, the small- and large- $x$ asymptotes of $y$ can be found already from (8.29) without knowing that $y=W(x)$. To this end, it suffices to recall the two basic features: (i) among all the elementary functions, $\ln x$ is the slowest to rise when its argument $x$ is augmented, and (ii) for a small value of its argument, the logarithmic function is large. Thus, for small $x$, the rhs of (8.29) is large. In the same limit $x \rightarrow 0$, the dominant term in the sum $y+\ln y$ from the lhs of (8.29) is $\ln y$. Thus, for small $x$, the relation (8.29) is reduced to $\ln y \approx \ln x$, which is $y \approx x$. On the other hand, for large $x$, the logarithmic function $\ln x$ on the rhs of (8.29) is large. In such a case, the lhs of (8.29) must also be large. When both $y$ and $\ln y$ are large, their sum $y+\ln y$ from (8.29) is dominated by $y$. Hence, for large $x$, the implicit equation (8.29) becomes $y \approx \ln x$. We see then that in the limits $x \rightarrow 0$ and $x \rightarrow \infty$ of the transcendental equation (8.29), the proper asymptotes of its solution $y$ via $y \approx x$ and $y \approx \ln x$, respectively, can faithfully be reconstructed with no recourse to the exact result (supposed here to be unknown) $y=W(x)$ of (8.29).

Similar asymptotic formulae can also be written for the Euler $T(x)$ function by using the defining relation $T(x) \mathrm{e}^{-T(x)}=x$ in (4.33) as well as $T(x)=\ln T(x)-\ln x$ from (4.34). In particular, the asymptote of $T(x)$ at small $x$ can be deduced directly from (8.27) via $x \rightarrow-x$ and $-W(-x)=T(x)$ yielding:

$$
\left.\begin{array}{rl}
T(x) \underset{x \rightarrow 0}{\underset{\rightarrow}{\approx}} \frac{x}{1-x} \\
\underset{x \rightarrow 0}{\approx} x+x^{2}
\end{array}\right\} .
$$

In (8.27), the small $x$-asymptote $W(x) \approx x /(1+x)=[1 / 1]_{W}(x)$ is the first-order diagonal Padé approximant ${ }^{1}$ (PA) to $W(x)$. The subsequent approximation $x /(1+$ $x) \approx x-x^{2}$ from (8.27) is obtained from the binomial expansion $1 /(1+x) \approx$ $1-x+x^{2}-\cdots(|x|<1)$ and by retaining only the first 2 terms $1-x$ to give $W(x) \approx x /(1+x) \approx x(1-x)=x-x^{2}$. If $x$ is a complex variable $z$, the asymptote of all the $k$ branches of the Lambert function $W_{k}(z)$ at large $|z|$ is given by:

\footnotetext{
1 Recall that for a series expansion in powers of $x$ of a given function $f(x)$ and for the fixed $(n, m)$ order, the PA to $f(x)$ is defined as the unique ratio $P_{n}(x) / Q_{n}(x)$ of two polynomials $P_{n}(x)$ and $Q_{m}(x)$ of degrees $n$ and $m$, respectively, as symbolized by $[n / m]_{f}(x)$, such that $f(x) \approx P_{n}(x) / Q_{n}(x)[111]$.
} 


$$
\left.\begin{array}{c}
\left.W_{k}(z)\right)_{|z|} \underset{\sim}{\approx} \operatorname{Ln} z-\ln (\operatorname{Ln} z) \\
W_{k}(z)_{|z|} \underset{\sim}{\approx} \infty \ln z+2 \pi i k-\ln (\ln z+2 \pi i k) \\
W_{k}(z)_{|z|} \underset{\rightarrow}{\approx} \ln \frac{z}{\ln z+2 \pi i k}+2 \pi i k
\end{array}\right\} \quad(k=0, \pm 1, \pm 2, \ldots) .
$$

\section{Applications of the Euler $T$ and Lambert $W$ functions in radiobiological modelings}

Dose planning systems in radiotherapy for treatment of patients with cancer rely heavily upon radiobiology. The problem of cell survival after dose exposure from different radiation modalities is one of the important themes of cell biology within radiobiology. Therein, a large number of mathematical models, both empirical and mechanistic, have been introduced over a period longer that 70 years. A partial account of the abundant bibliography can be found in a recent survey on this topic [3].

Radiation damage is inflicted on the healthy and diseased cells alike. The genetic system of normal cells can, in principle, repair at least some of their radiation lesions. However, in particular for cancerous cells, the repair system is dysfunctional due to their broken genetic machinery. Alternative to instantaneous doses, in order to follow the time course of cell recovery, fractionated dose exposures are also used for cell cultures (cell lines). This strategy is also administered for patients with cancers within the fractionated radiotherapy. With dose fractionation, cell recovery is thought to occur when cell survival after exposures to two doses separated in time (the two fractionated doses) is observed as being systematically larger than the corresponding cell survival following a single dose imparted at any time onto the treated cells.

For realistic radiobiological models of cell survival, in the case of both instantaneous and fractionated dose deliveries, cell recovery is one of the key effects that need to be properly taken into account. Metabolic repair processes can occur through various pathways that can be described by different mechanisms. Several among these mechanisms involving the Euler and Lambert functions have recently been put forward [1-3], and they will succinctly be outlined in the analysis which follows.

These advances in radiobiological models have been motivated by the need of dose planning systems for radiotherapy with hypo-fractionation which uses high doses [413]. The most frequently used radiobiological formalism, in all the dose planning systems is the LQ model [14-23] with the cell surviving fraction:

$$
S_{\mathrm{F}}^{(\mathrm{LQ})}(D)=\mathrm{e}^{-\alpha D-\beta D^{2}}, \quad \alpha>0, \quad \beta>0 .
$$

where the instantaneous dose is denoted by $D$. The cell survival curve drawn as the function $S_{\mathrm{F}}^{(\mathrm{LQ})}(D)$ versus dose keeps on bending as $D$ increases due to the presence of the Gaussian function $\exp \left(-\beta D^{2}\right)$ in $(9.1)$. Therefore, $S_{\mathrm{F}}^{(\mathrm{LQ})}(D)$ does not possess the correct high-dose behavior, which should be $S_{\mathrm{F}}(D) \approx \exp \left(-D / D_{0}\right)$, where $D_{0}$ is the mean lethal dose. This is mitigated in the LQL model whose biologically modified dose connects, at a transition dose $D_{\mathrm{T}}$, the LQ term $\alpha D+\beta D^{2}$ to a linear high-dose tail [26-36]. The resulting cell surviving fraction in the LQL model is: 


$$
\begin{aligned}
S_{\mathrm{F}}^{(\mathrm{LQL})}(D) & =\mathrm{e}^{-\alpha D-\beta D^{2}}, \quad D<D_{\mathrm{T}} \\
& =\mathrm{e}^{-\alpha D_{\mathrm{T}}-\beta D_{\mathrm{T}}^{2}-\gamma\left(D-D_{\mathrm{T}}\right)}, \quad D \geq D_{\mathrm{T}} .
\end{aligned}
$$

This deals with a discontinuous surviving fraction and, moreover, it has twice more free parameters $\left\{\alpha, \beta, \gamma, D_{\mathrm{T}}\right\}$ than the pair $\{\alpha, \beta\}$ in the LQ model. As will be evidenced by the upcoming exposition, it is much more rewarding to use the continuous surviving fractions, uniformly valid at all doses, within the recently introduced mechanistic threeparameter radiobiological models, based upon different mechanisms from chemical kinetics for cell repair of radiation damage [1-3].

\subsection{Integrated Michaelis-Menten method for cell survival}

Chemical kinetics can be viewed as one of the most appropriate ways to introduce repair mechanisms into cell survival after irradiation. Thus, for example, a cell repair process can be described through chemical kinetics by the irreversible enzyme catalysis within the formalism of the Michaelis-Menten equation [112] in the quasi-steady state (QSS) setting [113]. This has recently been done in Ref. [1]. Enzyme catalysis $[112,114]$, as one of the most important chemical reactions, consists of formation and destruction of an intermediate molecular compound:

$$
[\mathrm{E}]+[\mathrm{L}] \underset{k_{1}}{\longrightarrow}[\mathrm{EL}] \underset{k_{2}}{\longrightarrow}[\mathrm{E}]+[\mathrm{R}]
$$

where $[\mathrm{E}],[\mathrm{L}]$ and $[\mathrm{R}]$ are the concentrations of enzymes (E), lesions (L) \{ substrates and repaired lesions $(\mathrm{R})$ \{products , respectively. The concentration [EL] refers to the intermediate complex EL consisting of $\mathrm{E}$ and $\mathrm{L}$.

In biochemistry, the substrate and product are denoted by $\mathrm{S}$ and $\mathrm{P}$, respectively. This standard notation is not used in radiobiology to avoid confusion with the cell surviving fraction, which is conventionally labeled by $S$ or $S_{F}$. In (9.3), the parameters $k_{1}$ and $k_{2}$ are the rate constants for formation and destruction of EL, respectively. The damaged molecules (lesions L) can be taken to be the deoxyribonucleic acid (DNA) molecules, as the principal target in the cells. The products $\mathrm{R}$ are the repaired lesions.

In the Michaelis-Menten mechanism of enzyme catalysis for lesion repair, the mass action law for [E], [L], [EL] and [R] is employed through the standard system of differential non-linear coupled rate equations for time evolution of concentrations of the invoked four molecules:

$$
\begin{aligned}
& \frac{\mathrm{d}[\mathrm{L}]}{\mathrm{d} t}=-k_{1}[\mathrm{E}][\mathrm{L}], \\
& \frac{\mathrm{d}[\mathrm{E}]}{\mathrm{d} t}=-k_{1}[\mathrm{E}][\mathrm{L}]+k_{2}[\mathrm{EL}], \\
& \frac{\mathrm{d}[\mathrm{EL}]}{\mathrm{d} t}=k_{1}[\mathrm{E}][\mathrm{L}]-k_{2}[\mathrm{EL}], \\
& \frac{\mathrm{d}[\mathrm{R}]}{\mathrm{d} t}=k_{2}[\mathrm{EL}],
\end{aligned}
$$


with the given initial conditions at time $t=0$,

$$
[\mathrm{L}](0)=[\mathrm{L}]_{0}, \quad[\mathrm{E}](0)=[\mathrm{E}]_{0}, \quad[\mathrm{EL}](0)=[\mathrm{EL}]_{0}, \quad[\mathrm{R}](0)=[\mathrm{R}]_{0} .
$$

Hereafter, we use the abbreviated notation $[\mathrm{E}] \equiv[\mathrm{E}](t),[\mathrm{L}] \equiv[\mathrm{L}](t),[\mathrm{EL}] \equiv[\mathrm{EL}](t)$ and $[\mathrm{R}] \equiv[\mathrm{R}](t)$. The initial concentration of lesions $[\mathrm{L}]_{0}$ is:

$$
[\mathrm{L}]_{0}=k_{0} D, \quad k_{0}=\frac{1}{D_{0}},
$$

where as throughout, $D$ is the absorbed instantaneous (acute) physical dose. The quantity $D_{0}$ from (9.9) is the mean lethal dose, which is the dose for which the survival fraction is reduced by a factor of $1 / \mathrm{e} \approx 0.37$, or by $\sim 37 \%$. This follows from the assumption of a purely exponential decay law for the cell survival probability, $\mathrm{S}_{\mathrm{F}}(D)=\exp \left(-D / D_{0}\right)$, where at $D=D_{0}$ we have $S_{\mathrm{F}}\left(D_{0}\right)=1 / \mathrm{e}$.

The system of equations in (9.4)-(9.7) cannot be solved exactly by analytical means. However, making the QSS approximation, defined by $\mathrm{d}[\mathrm{EL}] / \mathrm{d} t \approx 0$, the exact closedform solution of the system (9.4)-(9.7) is obtained as the Michaelis-Menten equation for the reaction velocity $v_{0}[1]$ :

$$
v_{0} \equiv \frac{\mathrm{dR}}{\mathrm{d} t}=-\frac{\mathrm{dL}}{\mathrm{d} t}=\frac{v_{\max }[\mathrm{L}]}{K_{\mathrm{M}}+[\mathrm{L}]} .
$$

The parameter $v_{\max }>0$ is the maximal value of $v_{0}$ and $K_{\mathrm{M}}>0$ is the MichaelisMenten constant for the irreversible enzyme catalysis from (9.3):

$$
v_{\max }=k_{2}[\mathrm{E}]_{0}, \quad K_{\mathrm{M}}=\frac{k_{2}}{k_{1}} .
$$

The constant $K_{\mathrm{M}}$ has the dimension of concentration and it represents the value of the lesion concentration $[\mathrm{L}]$ at which the reaction velocity $v_{0}$ attains its halved maximum, $v_{\max } / 2$. Integrating the equation $\mathrm{dL} / \mathrm{d} t=-v_{\max }[\mathrm{L}] /\left(K_{\mathrm{M}}+[\mathrm{L}]\right)$ from $(9.10)$ gives the expression:

$$
-v_{\max } t=K_{\mathrm{M}} \ln \frac{[\mathrm{L}]}{[\mathrm{L}]_{0}}+[\mathrm{L}]-[\mathrm{L}]_{0} .
$$

An alternative derivation from Ref. [114] also gives the result (9.12), which for radiobiological models of cell surviving fractions has been used in Ref. [54]. It is convenient to rewrite Eq. (9.12) in the following equivalent form:

$$
\ln \frac{[\mathrm{L}]}{K_{\mathrm{M}}}+\frac{[\mathrm{L}]}{K_{\mathrm{M}}}=\ln y,
$$

with

$$
\ln y \equiv \ln \frac{[\mathrm{L}]_{0}}{K_{\mathrm{M}}}+\left\{\sigma_{\mathrm{M}}[\mathrm{L}]_{0}-k t\right\} \quad \therefore \quad y=\frac{[\mathrm{L}]_{0}}{K_{\mathrm{M}}} \mathrm{e}^{\sigma_{\mathrm{M}}[\mathrm{L}]_{0}-k t},
$$


where

$$
\sigma_{\mathrm{M}}=\frac{1}{K_{\mathrm{M}}}, \quad k=\frac{v_{\max }}{K_{\mathrm{M}}} .
$$

The implicit, transcendental equation (9.13) for [L], as a function of time $t$, can now be solved exactly using the definition (5.3) of the Lambert $W$ function with the result [1]:

$$
[\mathrm{L}](t)=\frac{1}{\sigma_{\mathrm{M}}} W_{0}\left(\sigma_{\mathrm{M}}[\mathrm{L}]_{0} \mathrm{e}^{\sigma_{\mathrm{M}}[\mathrm{L}]_{0}-k t}\right) .
$$

The Lambert function $W$ of a general argument $y$ from (5.3) is specified in (9.16) as the principal branch $W_{0}$ because $y=\left([\mathrm{L}]_{0} / K_{\mathrm{M}}\right) \exp \left(\sigma_{\mathrm{M}}[\mathrm{L}]_{0}-k t\right)$ from $(9.14)$ is always real and positive.

Earlier, in 1997, within biochemistry, the Michaelis-Menten equation has been integrated analytically for the first time by Schnell and Mendoza [88] in terms of the Lambert $W_{0}$ function. In 1999, Goudar et al. [89] applied intensively this analytical tool to biochemical problems, and made their program available as an open source code. From that time until the present, the Schnell-Mendoza method for integrated progress curves in the Michaelis-Menten based enzyme catalysis has continuously been applied for many problems in biochemistry and beyond.

From the radiobiological viewpoint, the compact analytical formula (9.16) is important because it depends both on time $t$ and dose $D$. Thus, the lesion concentration [L] $(t)$ is, in fact, a bi-variate function $[\mathrm{L}](t, D)$ which represents the net biological effect of radiation, as denoted by $\mathrm{E}_{\mathrm{B}}(t, D)$ :

$$
\mathrm{E}_{\mathrm{B}}(t, D) \equiv[\mathrm{L}](t, D)=\frac{1}{\sigma_{\mathrm{M}}} W_{0}\left(\sigma_{\mathrm{M}}[\mathrm{L}]_{0} \mathrm{e}^{\sigma_{\mathrm{M}}[\mathrm{L}]_{0}-k t}\right)
$$

However, whenever the dose dependence is of a primary concern, the time variable $t$ in (9.17) can be taken as a fixed time parameter. Presently, $t$ is taken to be the repair time $t_{\mathrm{R}}$. In such a case, and by reference to the relation $[\mathrm{L}]_{0}=k_{0} D$ from (9.9), dose $D$ becomes the sole independent variable in the lesion concentration $[\mathrm{L}]\left(t_{\mathrm{R}}, D\right)$, which is now a uni-variate function of dose, as simplified by $[\mathrm{L}]\left(t_{\mathrm{R}}, D\right) \equiv[\mathrm{L}](D)$, so that:

$$
\begin{aligned}
{[\mathrm{L}]\left(t_{\mathrm{R}}, D\right) } & \equiv[\mathrm{L}](D)=\frac{\alpha}{\gamma} W_{0}\left(\gamma D \mathrm{e}^{\beta D / \alpha}\right), \\
\alpha & =k_{0} \mathrm{e}^{-k t_{\mathrm{R}}}, \beta=k_{0} \gamma, \gamma=\frac{\alpha}{K_{\mathrm{M}}}, \\
\frac{\alpha}{\beta} & =K_{\mathrm{M}} D_{0}, \quad \frac{\gamma}{\beta}=D_{0} .
\end{aligned}
$$

As seen from (9.20), the parameter quotients $\alpha / \beta$ and $\beta / \gamma$ are independent on the repair time $t_{\mathrm{R}}$. This expounded formalism constitutes the integrated Michaelis-Menten radiobiolagical model, IMM [1] which, on account of (9.18), predicts the biological effect $\mathrm{E}_{\mathrm{B}}^{(\mathrm{IMM})}(D)$ as: 


$$
\mathrm{E}_{\mathrm{B}}^{(\mathrm{IMM})}(D)=\mathrm{L}^{(\mathrm{IMM})}(D)=\frac{\alpha}{\gamma} W_{0}\left(\gamma D \mathrm{e}^{\beta D / \alpha}\right) .
$$

Assuming the statistical Poisson distribution of lesions $\mathrm{L}^{(\mathrm{IMM})}(D)$, the associated cell surviving fraction $S_{\mathrm{F}}^{(\mathrm{IMM})}(D)$ in the IMM model is defined by $S_{\mathrm{F}}^{(\mathrm{IMM})}(D) \equiv$ $\exp \left\{-\mathrm{E}_{\mathrm{B}}^{(\mathrm{IMM})}(D)\right\}=\exp \left\{-\mathrm{L}^{(\mathrm{IMM})}(D)\right\}$ and, therefore:

$$
S_{\mathrm{F}}^{(\mathrm{IMM})}(D)=\mathrm{e}^{-(\alpha / \gamma) W_{0}\left(\gamma D \mathrm{e}^{\beta D / \alpha}\right)} .
$$

Using the asymptotic formulae (8.27) and (8.28), the behaviors of the surviving fraction $S_{\mathrm{F}}^{(\mathrm{IMM})}(D)$, for small and large doses, respectively, can readily be derived with the results [1]:

$$
\begin{gathered}
S_{\mathrm{F}}^{(\mathrm{IMM})}(D) \underset{D \rightarrow 0}{\approx} \mathrm{e}^{-D /\left(n D_{0}\right)}, \\
S_{\mathrm{F}}^{(\mathrm{IMM})}(D) \underset{D \rightarrow \infty}{\approx} n \mathrm{e}^{-D / D_{0}},
\end{gathered}
$$

where

$$
n=\mathrm{e}^{\omega_{\mathrm{R}}}, \quad \omega_{\mathrm{R}}=v_{\max } t_{\mathrm{R}}
$$

Here, $n$ is the extrapolation number, which is in the IMM model connected to the maximal enzyme velocity $v_{\max }$ and the repair time $t_{\mathrm{R}}$ via $\ln n=v_{\max } t_{\mathrm{R}}=\omega_{\mathrm{R}}$. In a plot of $S_{\mathrm{F}}^{(\mathrm{IMM})}(D)$ versus dose $D$, the number $n$ is the intercept of the extrapolated, ending, exponentially decreasing part of the survival curve with the ordinate (drawn from the origin $D=0$ ). The illustrations of the successful performance of the IMM model relative to measurements as well as to the LQ model for survival of irradiated cells have previously been given in Refs. [1,3].

\subsection{Cell repair by pool repair molecules from the cell environment}

Staying still within chemical kinetics, one of the alternatives to the IMM model is to conceive the damage repair as being mediated by the so-called pool repair molecules from the cell environment [56,115-120]. To proceed, let the set of quantities $\left\{[a]_{t},[b]_{t},[c]_{t},[p]_{t}\right\}$ be the concentrations of various types of radiation lesions $\{a, b, c, p\} \equiv\left\{{ }^{\prime \prime} a^{\prime \prime},{ }^{\prime \prime} b^{\prime \prime},{ }^{\prime \prime} c^{\prime \prime},{ }^{\prime \prime} p^{\prime \prime}\right\}$. Hereafter, in the running text, whenever the square brackets are not used to denote the concentrations, the letters $\{a, b, c, p\}$ for the lesion labels will alternatively be denoted by $\left\{{ }^{\prime \prime} a^{\prime \prime}, " b^{\prime \prime},{ }^{\prime \prime} c^{\prime \prime},{ }^{\prime \prime} p^{\prime \prime}\right\}$ for a clearer distinction from the corresponding ordinary letters. The meaning of the labels $[a]_{t},[b]_{t},[c]_{t}$ and $[p]_{t}$ is as follows: $[a]_{t}$ is the concentration of potentially lethal, first-step lesions per cell, $[b]_{t}$ is the concentration of metabolically-developed lethal lesions per cell, $[c]_{t}$ is the concentration of potentially lethal lesions per cell that have been repaired and $[p]_{t}$ is concentration of first-step lesions per cell that are not potentially lethal. Further, let $k_{0}, k, k_{1}, k_{2}$ be the rate constants of different transformations governed by chemical processes induced by interactions between the cell and radiation. Concretely, $k_{0}$ is 
the rate constant of increase in type " $a$ " lesions per unit dose at time $t=0, k$ is the constant of increase in type ${ }^{\prime \prime} p^{\prime \prime}$ lesions per unit dose at time $t=0, k_{1}$ is the rate constant of the cell kill reaction $[a]_{t} \longrightarrow[b]_{t}$ and $k_{2}$ is the rate constant for the recovery reaction $[a]_{t} \longrightarrow[c]_{t}$. Equivalently, rate $k_{0}$ can be defined through the reciprocal of the mean lethal dose $D_{0}$ via $k_{0}=1 / D_{0}$ as in (9.9).

Pool substances " $p$ " can be both repair molecules and lesions. The reason is that pool molecules can also be damaged by radiation, in which case, we assume that the produced " $p$ " lesions are not potentially lethal and are, thus, always available for repair. As stated, the rate constant $k_{2}$ is the rate for transformation " $a^{\prime \prime} \longrightarrow$ " $c^{\prime \prime}$ by which " $a$ " is reduced to " $c$ " per unit of " $a$ ". The rate $k_{1}$ of decrease of " $a^{\prime \prime}+{ }^{\prime \prime} p^{\prime \prime}$ can be assumed to be a dose-independent constant throughout the time development of the type " $a$ " lesions. The rate constant $k_{2}$ could be obtained by considering all lesions on which the recovery process can act. These are the type " $a$ " and " $b$ " lesions. A general hypothesis of all the pool-based models is that every increment of dose $D$ yields more new " $a$ " and " $p$ " lesions. Further, it can be supposed that the rate for the transformation " $a$ " $\longrightarrow$ " $b^{\prime \prime}$ is dependent solely on the concentration of the type " $a$ " lesions.

In this setting, the standard mass action law of chemical kinetics yields the following system of the rate equations (without forming an intermediate compound molecule):

$$
\begin{aligned}
\frac{\mathrm{d}[a]_{t}}{\mathrm{~d} t} & =-k_{2}[p]_{t}[a]_{t}-k_{1}[a]_{t}, \\
\frac{\mathrm{d}[b]_{t}}{\mathrm{~d} t} & =k_{1}[a]_{t}, \\
\frac{\mathrm{d}[c]_{t}}{\mathrm{~d} t} & =k_{2}[p]_{t}[a]_{t}, \\
\frac{\mathrm{d}[p]_{t}}{\mathrm{~d} t} & =-k_{2}[p]_{t}[a]_{t},
\end{aligned}
$$

with the initial conditions at $t=0$ :

$$
[a]_{0}=k_{0} D, \quad[b]_{0}=0, \quad[c]_{0}=0, \quad[p]_{0}=p_{0},
$$

where $p_{0}$ is the initial concentration of the pool molecules that are available for repair at time $t=0$. Unlike the Michaelis-Menten system of equations (9.4)-(9.7) for cell repair by enzyme catalysis, the system (9.26)-(9.29) can be solved exactly. To see this, we first express $[a]_{t}$ from Eq. (9.27) as:

$$
[a]_{t}=\frac{1}{k_{1}} \frac{\mathrm{d}[b]_{t}}{\mathrm{~d} t} .
$$

When (9.31) is inserted into Eq. (9.29), it follows:

$$
\frac{\mathrm{d} \ln [p]_{t}}{\mathrm{~d} t}=-\rho \frac{\mathrm{d}[b]_{t}}{\mathrm{~d} t},
$$


where $\rho$ is the quotient of the rate for the repair process and the development of a lethal lesion

$$
\rho=\frac{k_{2}}{k_{1}}=\frac{\text { Rate constant of cell repair }}{\text { Rate constant of cell kill }} .
$$

Integration of (9.32) gives $\ln [p]_{t}=-\rho[b]_{t}+\ln C_{1}$ where the integration constant is fixed as $C_{1}=p_{0}$ by the initial conditions (9.30). Thus:

$$
[p]_{t}=p_{0} \mathrm{e}^{-\rho[b]_{t}}
$$

Next, by combining (9.31) and (9.34), we can cast Eq. (9.28) for $[c]_{t}$ into the form:

$$
\frac{\mathrm{d}[c]_{t}}{\mathrm{~d} t}=\rho p_{0} \mathrm{e}^{-\rho[b]_{t}} \frac{\mathrm{d}[b]_{t}}{\mathrm{~d} t} .
$$

This equation has its more useful counterpart:

$$
\frac{\mathrm{d}[c]_{t}}{\mathrm{~d} t}=-p_{0} \frac{\mathrm{d}}{\mathrm{d} t} \mathrm{e}^{-\rho[b]_{t}}
$$

on the account of the relation

$$
\frac{\mathrm{d}}{\mathrm{d} t} \mathrm{e}^{-\rho[b]_{t}}=-\rho \mathrm{e}^{-\rho[b]_{t}} \frac{\mathrm{d}[b]_{t}}{\mathrm{~d} t} .
$$

Integration of Eq. (9.35) yields $[c]_{t}=-p_{0} \exp \left(-\rho[b]_{t}\right)+C_{2}$ with the integration constant $C_{2}$ determined as $C_{2}=p_{0}$ by way of the initial condition $[c]_{0}=0$ at $t=0$ from (9.30). Therefore:

$$
[c]_{t}=p_{0}\left\{1-\mathrm{e}^{-\rho[b]_{t}}\right\}
$$

Further, we insert (9.31) and (9.38) into the rhs of (9.26) to arrive at:

$$
\frac{\mathrm{d}[a]_{t}}{\mathrm{~d} t}=-\left\{1+\rho p_{0} \mathrm{e}^{-\rho[b]_{t}}\right\} \frac{\mathrm{d}[b]_{t}}{\mathrm{~d} t},
$$

or equivalently, with the help of (9.37)

$$
\frac{\mathrm{d}[a]_{t}}{\mathrm{~d} t}=-\frac{\mathrm{d}[b]_{t}}{\mathrm{~d} t}+p_{0} \frac{\mathrm{d}}{\mathrm{d} t} \mathrm{e}^{-\rho[b]_{t}} .
$$

Integration of this equation provides the result $[a]_{t}=-[b]_{t}+p_{0} \mathrm{e}^{-\rho[b]_{t}}+C_{3}$ where, by using the initial conditions $[a]_{0}=k_{0} D$ and $[b]_{0}=0$ from (9.30), the integration constant $C_{3}$ is found to be $C_{3}=k_{0} D-p_{0}$. This finally gives the expression:

$$
k_{0} D=[b]_{t}+p_{0}\left\{1-\mathrm{e}^{-\rho[b]_{t}}\right\}+[a]_{t}
$$


In the solution (9.41), the primary interest is to consider a sufficiently long time $t$ (theoretically $t \rightarrow \infty$ ). The corresponding limiting values of the invoked concentrations will be denoted by: $\lim _{t \rightarrow \infty}[a]_{t}=[a]_{\infty} \equiv[A]_{D}, \lim _{t \rightarrow \infty}[b]_{t}=[b]_{\infty} \equiv$ $[B]_{D}, \lim _{t \rightarrow \infty}[c]_{t}=[c]_{\infty} \equiv[C]_{D}, \lim _{t \rightarrow \infty}[p]_{t}=[p]_{\infty} \equiv[P]_{D}$. In particular, we have $[a]_{\infty}=0$ since after a sufficiently long time has elapsed, all the remaining potentially lethal lesions eventually lead to cell death. These circumstances map the expression (9.41) into the following relation:

$$
k_{0} D=[B]_{D}+p_{0}\left\{1-\mathrm{e}^{-\rho[B]_{D}}\right\},
$$

where the sought quantity $[B]_{D}$, as the biological effect of radiation, represents the concentration of lethal lesions at dose $D$. This implicit solution for $[B]_{D}$ belongs to the category of the following general transcendental equation:

$$
y-q_{1}-q_{2} \mathrm{e}^{-q_{3} y}=0 .
$$

Multiplying (9.43) by $q_{3} \mathrm{e}^{-q_{1} q_{3}}$ gives $q_{3}\left(y-q_{1}\right) \mathrm{e}^{-q_{1} q_{3}}=q_{2} q_{3} \mathrm{e}^{-q_{3}\left(y+q_{1}\right)}$ which can be rewritten as $q_{3}\left(y-q_{1}\right) \exp \left(q_{3}\left\{y-q_{1}\right\}\right)=q_{2} q_{3} \exp \left(-q_{1} q_{3}\right)$, or equivalently:

$$
Y \mathrm{e}^{Y}=q_{2} q_{3} \mathrm{e}^{-q_{1} q_{3}},
$$

where $Y$ is an abbreviated notation for $q_{3}\left(y-q_{1}\right)$

$$
Y=q_{3}\left(y-q_{1}\right)
$$

As per (5.2), the solution $Y$ of (9.44) is the Lambert function $Y=W\left(q_{2} q_{3} \mathrm{e}^{-q_{1} q_{3}}\right)$, so that after returning to $y$ via $(9.45)$, namely $y=q_{1}+\left(1 / q_{3}\right) Y$, it follows:

$$
y=q_{1}+\frac{1}{q_{3}} W\left(q_{2} q_{3} \mathrm{e}^{-q_{1} q_{3}}\right) .
$$

Rewriting (9.42) in the form (9.43) as $[B]_{D}-\left(k_{0} D-p_{0}\right)-p_{0} \mathrm{e}^{-\rho[B]_{D}}=0$ permits the identification:

$$
y=[B]_{D}, q_{1}=k_{0} D-p_{0}, q_{2}=p_{0}, q_{3}=\rho .
$$

These relations together with (9.46) give the solution of (9.42) as:

$$
[B]_{D}=k_{0} D-p_{0}+\frac{1}{\rho} W_{0}\left(\rho p_{0} \mathrm{e}^{-\rho\left(k_{0} D-p_{0}\right)}\right),
$$

where the principal branch $W_{0}$ of $W$ is taken because its independent variable is always non-negative, $\rho p_{0} \exp \left(-\rho\left(k_{0} D-p_{0}\right)\right) \geq 0$, for all the physical values of the invoked quantities $\left\{D ; \rho, k_{0}, p_{0}\right\}$. From here, the pool repair Lambert model, PRL [2] 
for the biological effect of radiation, as the concentration of lethal lesions, has been introduced by:

$$
\mathrm{E}_{\mathrm{B}}^{(\mathrm{PRL})}(D)=[B]_{D}=k_{0} D-p_{0}+\frac{1}{\rho} W_{0}\left(\rho p_{0} \mathrm{e}^{-\rho\left(k_{0} D-p_{0}\right)}\right) .
$$

With the usual assumption of a Poisson distribution of lesions $[B]_{D}$, the associated cell surviving fraction in the PRL model is:

$$
S_{\mathrm{F}}^{(\mathrm{PRL})}(D) \equiv \mathrm{e}^{-\mathrm{E}_{\mathrm{B}}^{(\mathrm{PRL})}(D)}=\mathrm{e}^{-\left(k_{0} D-p_{0}\right)-(1 / \rho) W_{0}\left(\rho p_{0} \mathrm{e}^{-\rho\left(k_{0} D-p_{0}\right)}\right)} .
$$

An alternative form of (9.43) can also be considered by the substitution:

$$
S=\mathrm{e}^{-y}
$$

With this ansatz, we can cast (9.43) into the following general transcendental equation:

$$
\ln S+q_{1}+q_{2} S^{q_{3}}=0
$$

or equivalently

$$
\frac{1}{q_{3}} \ln \left(q_{2}^{1 / q_{3}} S\right)^{q_{3}}-\frac{\ln \left(q_{2}\right)}{q_{3}}+q_{1}+\left(q_{2}^{1 / q_{3}} S\right)^{q_{3}}=0 .
$$

Further, passing from the function $S$ to $X$ by using the defining relation:

$$
X=q_{2} S^{q_{3}}
$$

it follows from (9.53)

$$
\ln X+q_{3} X=\ln \left(q_{2}\right)-q_{1} q_{3}
$$

or alternatively

$$
\ln Y+Y=\ln \zeta, \quad Y=q_{3} X
$$

where

$$
\ln \zeta=\ln \left(q_{2} q_{3}\right)-q_{1} q_{3} \quad \therefore \quad \zeta=q_{2} q_{3} \mathrm{e}^{-q_{1} q_{3}} .
$$

A comparison of (9.56) with (5.3) provides the following identification:

$$
Y=W(\zeta)
$$


This result, by recurring back to $X$, yields $X=\left(1 / q_{3}\right) W(\zeta)$. The latter formula, by way of (9.54), provides $S$ as:

$$
S=\left\{\frac{1}{q_{2} q_{3}} W\left(q_{2} q_{3} \mathrm{e}^{-q_{1} q_{3}}\right)\right\}^{1 / q_{3}} .
$$

This is the solution of the intermediate transcendental equation (9.52), which is of interest on its own right. Finally, by recuperating the original quantity $y$ from $S$ through the use of (9.51), we arrive at:

$$
y=-\frac{1}{q_{3}} \ln \left(\frac{1}{q_{2} q_{3}} W\left(q_{2} q_{3} \mathrm{e}^{-q_{1} q_{3}}\right)\right) .
$$

We now have two very differently looking expressions (9.46) and (9.60) for the supposedly unique solution $y$ of the starting transcendental equation (9.43). The solution $y$ from (9.43) would indeed be unique if and only if the right hand sides of the expressions in (9.46) and (9.60) are identical. To proceed, we then assume that $y$, as the solution of transcendental equation (9.43), is equal to either the left or the right hand sides of the following expression taken from (9.46) and (9.60):

$$
q_{1}+\frac{1}{q_{3}} W(\zeta)=-\frac{1}{q_{3}} \ln \left(\frac{W(\zeta)}{q_{2} q_{3}}\right)
$$

where $\zeta=q_{2} q_{3} \mathrm{e}^{-q_{1} q_{3}}$ as per (9.57). Multiplying both sides of Eq. (9.61) by $q_{3}$ and rearranging the resulting terms, we obtain $\ln (W(\zeta))+W(\zeta)=\ln \left(q_{2} q_{3}\right)-q_{1} q_{3}$. The rhs of this latter equation is equal to $\ln \zeta$, according to (9.57), so that:

$$
\ln W(\zeta)+W(\zeta)=\ln (\zeta)
$$

This is of the form of the definition (5.3) of the Lambert $W$ function. Therefore, the indicated match of the two different forms (9.46) and (9.60), as stated in (9.61), coherently recovers one of the defining relations for the $W$ function, namely Eq. (5.3). This completes the sought proof of the identity of the solutions (9.46) and (9.60) of the transcendental equation (9.43).

In the context of radiobiology, referring to (9.47), where $y$ is the biological effect $[B]_{D} \equiv \mathrm{E}_{\mathrm{B}}^{(\mathrm{PRL})}(D)$ predicted by the PRL model, the quantity $S$ from $(9.51)$ is identified as the cell surviving fraction $S_{\mathrm{F}}^{(\mathrm{PRL})}(D)$. Thus, with the parameters $\left\{q_{1}, q_{2}, q_{3}\right\}$ taken according to (9.47), in the outlined second derivation, the biological effect and the cell surviving fractions acquires the following alternative forms, that are equivalent to (9.48) and (9.50), respectively [2]:

$$
\mathrm{E}_{\mathrm{B}}^{(\mathrm{PRL})}(D)=-\frac{1}{\rho} \ln \left(\frac{1}{\rho p_{0}} W_{0}\left(\rho p_{0} \mathrm{e}^{-\rho\left(k_{0} D-p_{0}\right)}\right)\right),
$$


and

$$
S_{\mathrm{F}}^{(\mathrm{PRL})}(D)=\left\{\frac{1}{\rho p_{0}} W_{0}\left(\rho p_{0} \mathrm{e}^{-\rho\left(k_{0} D-p_{0}\right)}\right)\right\}^{1 / \rho} .
$$

The alternative result (9.64) must be equal to (9.50). To show this, we denote the argument $\rho p_{0} \exp \left\{-\rho\left(k_{0} D-p_{0}\right)\right\}$ of the $W_{0}$ function from (9.63) as $x_{D}$ via:

$$
x_{D} \equiv \rho p_{0} \mathrm{e}^{-\rho\left(k_{0} D-p_{0}\right)} \quad \therefore \quad \ln \left(x_{D}\right)=\ln \left(\rho p_{0}\right)-\rho\left(k_{0} D-p_{0}\right) \text {. }
$$

In terms of the quantity $x_{D}$, and by reference to (5.2) and (5.3), the function $W_{0}\left(x_{D}\right)$ from (9.64) reads as:

$$
W_{0}\left(x_{D}\right) \mathrm{e}^{W_{0}\left(x_{D}\right)}=x_{D} \quad \therefore \quad \ln W_{0}\left(x_{D}\right)+W_{0}\left(x_{D}\right)=\ln \left(x_{D}\right) .
$$

This is followed by taking the natural logarithm of both sides of (9.64):

$$
\begin{aligned}
\ln S_{\mathrm{F}}^{(\mathrm{PRL})}(D) & =\frac{1}{\rho} \ln \frac{W_{0}\left(x_{D}\right)}{\rho p_{0}} \\
& =\frac{1}{\rho} \ln W_{0}\left(x_{D}\right)-\frac{1}{\rho} \ln \left(\rho p_{0}\right) \\
& =\frac{1}{\rho}\left\{\ln \left(x_{D}\right)-W_{0}\left(x_{D}\right)\right\}-\frac{1}{\rho} \ln \left(\rho p_{0}\right) \\
& =-\frac{W_{0}\left(x_{D}\right)}{\rho}+\frac{1}{\rho}\left\{\ln \left(\rho p_{0}\right)-\rho\left(k_{0} D-p_{0}\right)\right\}-\frac{1}{\rho} \ln \left(\rho p_{0}\right) \\
& =-\left(k_{0} D-p_{0}\right)-\frac{W_{0}\left(x_{D}\right)}{\rho}
\end{aligned}
$$

so that

$$
\ln S_{\mathrm{F}}^{(\mathrm{PRL})}(D)=-\left(k_{0} D-p_{0}\right)-\frac{1}{\rho} W_{0}\left(\rho p_{0} \mathrm{e}^{-\rho\left(k_{0} D-p_{0}\right)}\right) .
$$

The result (9.67) coincides with (9.49), which itself is equal to $\ln S_{\mathrm{F}}^{(\mathrm{PRL})}(D)$. This proves the equivalence of (9.50) and (9.64). Both forms for the PRL model, i.e. (9.50) and (9.64) have only three parameters $\left\{k_{0}, p_{0}, \rho\right\}$ and they all possess their mechanistic meaning derived from chemical kinetics [2].

Regarding the low- and high- $D$ asymptotes of the cell surviving fraction $S_{\mathrm{F}}^{(\mathrm{PRL})}(D)$ from (9.50), they can be established, as has been done in Ref. [2], in a way similar to that presently explained for the IMM model. Thus, the corresponding asymptotic behaviors of $S_{\mathrm{F}}^{(\mathrm{PRL})}(D)$ are given by:

$$
\begin{aligned}
& S_{\mathrm{F}}^{(\mathrm{PRL})}(D) \underset{D \rightarrow 0}{\approx} \mathrm{e}^{-D /\left\{\left(1+\rho p_{0}\right) D_{0}\right\}}, \\
& S_{\mathrm{F}}^{(\mathrm{PRL})}(D) \underset{D \rightarrow \infty}{\approx} n \mathrm{e}^{-D / D_{0}},
\end{aligned}
$$


where $n$ is the extrapolation number

$$
n=\mathrm{e}^{p_{0}} .
$$

In the PRL model, the extrapolation number $n$ from (9.70) is different from its counterpart (9.25) in the IMM model. Namely, $n$ from (9.70) in the PRL model is defined in terms of the initial concentration $p_{0}$ of the pool repair molecules. Otherwise, the meaning of $n$ in a plot of $S_{\mathrm{F}}^{(\mathrm{PRL})}(D)$ as a function of dose $D$, is the same as that already explained in the IMM model. The PRL model has been shown to excellently reproduce the experimental data on cell surviving fractions at all radiation doses $D$ ranging from low through intermediate to the highest radiation exposures [2].

\subsection{The Euler $T$ function for corrections to counting statistics}

As an apparent digression from the theme of the applications of the Euler and Lambert functions in radiobiology, let us mention a circumstance associated with radiation detectors. Namely, it is a common occurrence that the observed counting rates in radiation detecting instruments are only apparent and, as such, they need the so-called counting coincidence correction. In these measurements, counters take their time (say $\tau$ ) to "recover" from radiation hits. The meaning of such a notion is that once a count is recorded, the counting detector becomes insensitive during a time $\tau$. As a consequence of the inequality $\tau \neq 0$, the detector cannot register all the hits. Therefore, the observed number of counts will only be apparent as it is smaller than the true number of radiation hits. In other words, there is a delay in counting, yielding a measurement error due to the missed (non-registered) hits. So the practical question arises as to how to correct the experimental counting rate $n_{\exp }$ to deduce the corresponding expected, true counting rate $n_{\text {true }}$. The posed question can be answered in an adequate manner by assuming the Poisson statistics of random events. Such a distribution gives for the corrected count rates (say $n_{\text {corr }}$ ) the following result [3]:

$$
n_{\text {corr }}=n_{\exp } \mathrm{e}^{-n_{\exp } \tau}
$$

which is of the type of the Ricker decline function $y=x \mathrm{e}^{-a x}$ from (4.1) in population dynamics [104]. This is the source of the so-named updating (extendable, or paralyzable or cumulative) dead time model in counting statistics. The formula (9.71) is universally useful for a variety of coincidence counting experimental techniques that need not refer to radiation measurements. Employing (9.71), the true counts $n_{\text {true }}$ can be modeled by $n_{\text {corr }}$, which corrects for the dead time $\tau$ of detector. The sought corrected counting rate $n_{\text {corr }}$ is reconstructed from (9.71) as the inverse function by means of the definition (4.32) for the Euler $T$ function. This is already stated in (4.13) for the function (4.1) which reduces to (9.71) for $a=\tau, x=n_{\exp }$ and $y=n_{\text {corr }}$, so that:

$$
n_{\mathrm{corr}}=\frac{1}{\tau} T_{0}\left(n_{\exp } \tau\right)
$$


With the help of (4.14), the result (9.72) can be expressed by the following series expansion in powers of the measured counting rates $n_{\exp }$ :

$$
\begin{aligned}
n_{\mathrm{corr}} \tau & \equiv \sum_{n=1}^{\infty} \frac{n^{n-1}}{n !}\left(n_{\exp } \tau\right)^{n} \\
& =\xi+\xi^{2}+\frac{3}{2} \xi^{3}+\frac{8}{3} \xi^{4}+\frac{125}{24} \xi^{5}+\frac{54}{5} \xi^{6}+\frac{16807}{720} \xi^{7}+\cdots
\end{aligned}
$$

where $\xi=n_{\exp } \tau$. The exact solution (9.72) of the transcendental equation (9.71) in counting statistics of radiation detectors has been obtained in Ref. [3] explicitly and directly through the Euler $T_{0}$ function. The series in (9.73) for the cumulative dead time has been derived earlier in Ref. [121] using the method of series inversion. However, it was not realized in Ref. [121] that (9.73) coincides with the the power series representation of the Euler $T_{0}$ function. Needless to say, it is of theoretical and practical importance to recognize that the rhs of Eq. (9.73) is the Euler $T_{0}$ function as this paves the road for exploration of various properties of this function, e.g. its asymptotic forms, etc.

\subsection{The Euler $T$ function stemming from counting statistics for cell survival}

The subject of the Sect. 9.3 is of direct relevance to a key concept of the so-called biologically effective dose. This concept is rooted in the mechanism of cell repair. Viewed as a counting "detector", the cell can be considered as having its resolving power. In this way, the cell could, in principle, separate any two consecutive hits on its sensitive site targeted by radiation. Namely, for any time $t$ smaller than the cell repair (recovery) time $\tau$, the cell would be insensitive to radiation. In other words, such a cell radiation insensitivity acts as if the cell were blocked to all the hits at the times $t<\tau$. This cell blocking mechanism (as a kind of radiation shielding), with the underlying time delay, amounts to reduction of the effectiveness of radiation. As a consequence, the cell absorbs a dose $D_{\mathrm{B}}$ which is decreased relative to the delivered instantaneous dose $D$. The latter relation, $D_{\mathrm{B}}<D$, as a manifestation of a reduced radiosensitivity of the cell, conceives the dose $D_{\mathrm{B}}$ as a biological modification of the imparted physical dose $D$. Thus, the cell blocking mechanism, facilitated by the existence of the cell repair system, alters $D$ to $D_{\mathrm{B}}$ as if the cell were annihilating all the radiation quanta that were supposed to hit the targeted cell at the times $t<\tau$.

These remarks resonate with the coincidence counting correction from the Sect. 9.3 and, moreover, they can make the relation $D_{\mathrm{B}}<D$ quantitative. To proceed, we again assume the Poisson statistics to formalize the effect of the missed or wasted radiation quanta. According to this assumption, the probability $P(0)$ of having no radiation events at times $t<\tau$ is given by $P(0)=\exp (-k \tau)=\exp \left(-\mu D_{\mathrm{B}}\right)$. Here, $k$ and $\mu$ are, respectively, the dose-rate-dependent and dose-rate independent constants for producing sub-lethal lesions by radiation. The same probability $P(0)$ quantifies the mentioned relation $D_{\mathrm{B}}<D$ as $D_{\mathrm{B}} P(0)=D$. This gives the way in which the dose $D$ is decreased to $D_{\mathrm{B}}$ by the cell repair system mediated through the cell blocking mechanism in the setting of the Poisson distribution of lesions [47-53]: 


$$
D_{\mathrm{B}} \mathrm{e}^{-\mu D_{\mathrm{B}}}=D, \quad \mu>0
$$

If we take $D$ to be the function of the given independent variable $D_{\mathrm{B}}$, then (9.74) is the Ricker decline function from (4.1). However, in the context of inverse functions, $D$ (dependent variable) is given and $D_{\mathrm{B}}$ is the sought function (independent variable). Using the definition (4.33) of the Euler $T$ function, the biologically modified dose $D_{\mathrm{B}}$ can be retrieved from $(9.74)$ as:

$$
D_{\mathrm{B}}=\frac{1}{\mu} T_{0}(\mu D),
$$

or via the equivalent power series representation

$$
\begin{aligned}
D_{\mathrm{B}}= & \frac{1}{\mu} T_{0}(\mu D) \\
= & \sum_{n=1}^{\infty}(n \mu)^{n-1} \frac{D^{n}}{n !}=\left(D+\mu D^{2}\right) \\
& +\frac{3}{2} \mu^{2} D^{3}+\frac{8}{3} \mu^{3} D^{4}+\frac{125}{24} \mu^{4} D^{5}+\frac{54}{5} \mu^{5} D^{6}+\frac{16807}{720} \mu^{6} D^{7}+\cdots
\end{aligned}
$$

As per the explanation, the cell-blocking mechanism effectively relies upon the existence of a time delay $\Delta t$ due to the cell's inability to register any hit at times $t$ smaller than $\Delta t$. Here, $\Delta t$ is the same as the cell repair time $\tau$, i.e. $\Delta t=\tau$. For this reason, modeling the biologically modified dose by means of $D_{\mathrm{B}}$ from (9.75) in the cell surviving fraction $S_{\mathrm{F}}(D) \equiv \exp \left(-\alpha D_{\mathrm{B}}\right)$ is called the Euler delayed dynamics (EDD) model [47-52]. Thus, writing $S_{\mathrm{F}}^{(\mathrm{EDD})}(D) \equiv \exp \left\{-\alpha D_{\mathrm{B}}^{(\mathrm{EDD})}\right\}$ with $D_{\mathrm{B}}$ from (9.77) re-labeled by $D_{\mathrm{B}}^{(\mathrm{EDD})}$, we have:

$$
S_{\mathrm{F}}^{(\mathrm{EDD})}(D)=\mathrm{e}^{-(\alpha / \mu) T_{0}(\mu D)}
$$

If we set $\mu=\beta / \alpha$ and truncate the series for $D_{\mathrm{B}}$ by retaining only the first two terms $D+\mu D^{2}$, that are already singled out in (9.76), the LQ model would be obtained for the biological effect $\alpha D_{\mathrm{B}} \approx \alpha D_{\mathrm{B}}^{(\mathrm{LQ})}=\alpha D+\beta D^{2}$. This would give the cell surviving fraction $S_{\mathrm{F}}^{(\mathrm{LQ})}(D)=\exp \left(-\alpha D-\beta D^{2}\right)$, as in (9.1). As such the LQ model appears to be a low-dose approximation of the EDD model.

\subsection{The Euler $T$ function in cell survival stemming explicitly from delayed dynamics}

The EDD model can alternatively be derived in the framework of delayed differential equations (DDE) [3]. To see this, instead of using the time variable, we will temporarily consider the radiosensitivity $\alpha$ as the independent variable, and take dose $D$ to be a fixed parameter. Note that in the simplest radiobiological model $S_{\mathrm{F}}(\alpha, D)=\mathrm{e}^{-\alpha D}=$ 
$S_{\mathrm{F}}(D, \alpha)$, the quantities $\alpha$ and $D$ appear symmetrically. This implies that the same solution $S_{\mathrm{F}}(D)=\mathrm{e}^{-\alpha D}$ of an underlying dynamical equation can be derived by exchanging the role of $\alpha$ and $D$. For example, we can use $D$ as the independent variable and keep $\alpha$ as a fixed, constant parameter. This situation corresponds to the cell dynamics described by the ordinary differential equation (ODE):

$$
\left.\begin{array}{c}
\frac{\mathrm{d} S_{\mathrm{F}}(\alpha, D)}{\mathrm{d} D}=-\alpha S_{\mathrm{F}}(\alpha, D), \quad S_{\mathrm{F}}(\alpha, 0)=1 \\
\therefore \quad S_{\mathrm{F}}(\alpha, D)=\mathrm{e}^{-\alpha D}
\end{array}\right\}, \quad \mu>0 .
$$

Likewise, we can take $\alpha$ as the independent variable and consider $D$ as a fixed parameter, so that the alternative ODE is:

$$
\left.\begin{array}{c}
\frac{\mathrm{d} S_{\mathrm{F}}(\alpha, D)}{\mathrm{d} \alpha}=-D S_{\mathrm{F}}(\alpha, D), \quad S_{\mathrm{F}}(0, D)=1 \\
\therefore \quad S_{\mathrm{F}}(\alpha, D)=\mathrm{e}^{-\alpha D}
\end{array}\right\}
$$

Both Eqs. (9.79) and (9.80) describe an irreparable lesion mediated by a direct cell kill mechanism. Additionally, repair can be included by introducing a time delay $\tau$ which retards the direct radiation action. Such a type of delayed dynamics is described by a DDE, which usually has time $t$ as the independent variable. This does not need to be always the case, and in the present context, we can use the radiosensitivity $\alpha$ as the independent variable. Under such a circumstance, to introduce a cell repair mechanism, we shall consider the following DDE counterpart of the ODE from (9.80):

$$
\frac{\mathrm{d} S_{\mathrm{F}}(\alpha, D)}{\mathrm{d} \alpha}=-f(D) S_{\mathrm{F}}(\alpha-\mu, D), S_{\mathrm{F}}(0, D)=1, \quad \mu>0
$$

where $D$ and $\mu$ are fixed non-negative parameters. In particular, $\mu$ is a delay constant which is a reduction of radiosensitivity in comparison with to the situation without repair. Here, $f(D)$ is a dose-modifying factor (DMF), which is subject to various choices leading to different radiobiological models. To solve Eq. (9.81), we set:

$$
S_{\mathrm{F}}(\alpha, D)=\mathrm{e}^{\alpha X_{D}}
$$

The sought function $X_{D}$ does not depend on $\alpha$. Its dependence on $D$ is implicit and parametric. When the ansatz (9.82) is substituted in Eq. (9.81), it follows $X_{D} \mathrm{e}^{\alpha X_{D}}=$ $-f(D) \mathrm{e}^{(\alpha-\mu) X_{D}}$, or equivalently:

$$
X_{D} \mathrm{e}^{\mu X_{D}}=-f(D)
$$

Multiplication by $\mu$ gives the transcendental equation $\mu X_{D} \mathrm{e}^{\mu X_{D}}=-\mu f(D)$ whose exact solution, according to (5.1) and (5.2), is the Lambert $W$ function, i.e. $\mu X_{D}=$ $W(-\mu f(D))$. This latter expression can equivalently be given through the Euler $T$ function via $\mu X_{D}=-T(\mu f(D))$, by virtue of the relation $W(-y)=-T(y)$ from (3.6), so that: 


$$
X_{D}=-\frac{1}{\mu} T(\mu f(D)) .
$$

Inserting this result into Eq. (9.82) gives the cell survival fraction:

$$
S_{\mathrm{F}}(\alpha, D)=\mathrm{e}^{-(\alpha / \mu) T(\mu f(D))} .
$$

Once (9.85) is derived, we are free to return to the usual writing via $S_{\mathrm{F}}(D)$ for the cell surviving fraction instead of the provisional notation $S_{\mathrm{F}}(\alpha, D)$. Then, for a specified $f(D)$, the surviving fraction $S_{\mathrm{F}}(D)$, as a function of $D$, can be drawn with the quantity $\alpha$ considered as a fixed parameter. For example, we can model the DMF to be linear in dose $D$ :

$$
f(D)=D .
$$

In this case, $T(\mu f(D))$ from (9.85) becomes the Euler principal branch function $T_{0}(\mu D)$ since $\mu D$ is positive. With $f(D)$ given by (9.86), the surviving fraction (9.85) becomes:

$$
S_{\mathrm{F}}(D)=\mathrm{e}^{-(\alpha / \mu) T_{0}(\mu f(D))}=\mathrm{e}^{-(\alpha / \mu) T_{0}(\mu D)}, \quad f(D)=D,
$$

where we set $S_{\mathrm{F}}(\alpha, D) \equiv S_{\mathrm{F}}(D)$. This expression coincides with the surviving fraction $S_{\mathrm{F}}^{(\mathrm{EDD})}(D)$ from Eq. (9.78) in the EDD model. Of course, in the DDE formalism, the parameter $\mu$ must be independent of $\alpha$ to secure that $S_{\mathrm{F}}(D)$ from (9.87) is the exact solution of Eq. (9.81).

\subsection{The Euler $T$ function for cell survival based upon delayed dynamics and the Poisson dose modifying factor}

As stated, in the DDE from (9.81), the function $f(D)$ is not specified. As such, the choice (9.86) produced the EDD model. Of course, some other biologically justified choices of the DMF could also be made. For example, we can request that $f(D)$ is given by the Poissonian ansatz via the Ricker decline function (3.3):

$$
f(D)=D \mathrm{e}^{-\lambda D}, \quad \lambda>0,
$$

where $\lambda$ is a repair rate constant. Note that the rhs of Eq. (9.88) would correspond to the cell blocking mechanism of repair, if in Eq. (9.74), the parameter $\mu$ is renamed to be $\lambda$ alongside with the exchange $D_{\mathrm{B}} \longleftrightarrow D$. Inserting (9.88) into (9.85) gives the presently proposed Euler-Poisson Delayed (EPD) model with the surviving fraction:

$$
S_{\mathrm{F}}^{(\mathrm{EPD})}(D)=\mathrm{e}^{-(\alpha / \mu) T_{0}\left(\mu D \mathrm{e}^{-\lambda D}\right)}, \quad f(D)=D \mathrm{e}^{-\lambda D} .
$$

The dose-modifying factor $f(D)$ from (9.86) in the EDD model does not, in fact, modify the imparted dose $D$, i.e. $f(D)=D$. However, in the EPD model, the situation 
is different. Therein, the dose-modifying factor $f(D)$ from (9.88) relates to a manner by which the repair system copes with the impact of radiation. Namely, by way of $f(D)=D \exp (-\lambda D)$ from (9.88), the cell repair capability is significant for small doses, after which it reaches a maximum at $D=1 / \lambda$ and afterward, as expected, dies out exponentially at high doses.

\subsection{The Lambert $W$ function for cell survival based upon delayed dynamics}

Within the delayed dynamics, another radiobiological model can be devised. To this end, it is convenient to first consider an auxiliary function $M(\alpha, D)$, called the cell mortality function, which is defined by the following DDE:

$$
\frac{\mathrm{d} M(\alpha, D)}{\mathrm{d} \alpha}=g(D) M(\alpha-\mu, D), \quad M(0, D)=1,
$$

where $\mu>0$ and $D$ is a fixed parameter. Here, $g(D)$ is a growth function, which can be selected following different models from studies of population species. Then the reciprocal of $M(\alpha, D)$ is the surviving fraction $S_{\mathrm{F}}(\alpha, D)$ via:

$$
S_{\mathrm{F}}(\alpha, D)=\frac{1}{M(\alpha, D)}
$$

In Eq. (9.90), we can choose $g(t)$ to be the Ricker growth function of the type (3.1), as proposed in Ref. [3]:

$$
g(D)=D \mathrm{e}^{\lambda D}, \quad \lambda>0
$$

With this choice, Eq. (9.90) becomes:

$$
\frac{\mathrm{d} M(\alpha, D)}{\mathrm{d} \alpha}=D \mathrm{e}^{\lambda D} M(\alpha-\mu, D), \quad M(0, D)=1
$$

The solution of (9.93) can be found by introducing the following exponential ansatz:

$$
M(\alpha, D)=\mathrm{e}^{\alpha Y_{D}}
$$

Substituting this form of $M(\alpha, D)$ into (9.93) yields the expression:

$$
Y_{D} \mathrm{e}^{\alpha Y_{D}}=D \mathrm{e}^{\lambda D+(\alpha-\mu) Y_{D}}
$$

Multiplication of both sides of this equation by $\mu \mathrm{e}^{(\mu-\alpha) Y_{D}}$ gives:

$$
\mu Y_{D} \mathrm{e}^{\mu Y_{D}}=\mu D \mathrm{e}^{\lambda D}
$$


This transcendental equation is solved in terms of the principal value Lambert $W_{0}$, since $\mu D \exp (\lambda D)$ is positive:

$$
Y_{D}=\frac{1}{\mu} W_{0}\left(\mu D \mathrm{e}^{\lambda D}\right)
$$

Therefore, the cell mortality function $M(\alpha, D)$ from (9.94) acquires the form:

$$
M(\alpha, D)=\mathrm{e}^{(\alpha / \mu) W_{0}\left(\mu D \mathrm{e}^{\lambda D}\right)},
$$

and the ensuing result for the quantity $S_{\mathrm{F}}(\alpha, D)$ from (9.91) becomes

$$
S_{\mathrm{F}}(\alpha, D)=\mathrm{e}^{-(\alpha / \mu) W_{0}\left(\mu D \mathrm{e}^{\lambda D}\right)} .
$$

In an analogy with the EDD model from the formalism of the DDEs, here too, the function $S_{\mathrm{F}}(\alpha, D)$ from (9.99) can freely be re-labeled as $S_{\mathrm{F}}(D)$ to represent the cell surviving fraction in which $\alpha$ is now kept as a fixed, constant parameter. The outlined description of the delayed dynamics resulting in the biological effect expressed in terms of the Lambert function $W_{0}\left(\mu D \mathrm{e}^{\lambda D}\right)$ is called the Lambert delayed dynamics (LDD) model [3]:

$$
S_{\mathrm{F}}^{(\mathrm{LDD})}(D)=\mathrm{e}^{-(\alpha / \mu) W_{0}\left(\mu D \mathrm{e}^{\lambda D}\right)} .
$$

The LDD model provides the analytical expressions for the required asymptotic behaviors of the surviving fraction $S_{\mathrm{F}}^{(\mathrm{LDD})}(D)$. It predicts the correct exponential cell inactivation modes at both small and large doses with a shoulder located in between these two extreme ranges of irradiation.

Formally, the cell surviving fractions $S_{\mathrm{F}}^{(\mathrm{IMM})}(D)$ and $S_{\mathrm{F}}^{(\mathrm{LDD})}(D)$ from $(9.22)$ and (9.100) in the IMM and LDD models, respectively, are strikingly similar. As a matter of fact, they would coincide via $S_{\mathrm{F}}^{(\mathrm{IMM})}(D)=S_{\mathrm{F}}^{(\mathrm{LDD})}(D)$ if the parameters $\mu$ and $\lambda$ from $S_{\mathrm{F}}^{(\mathrm{LDD})}(D)$ in (9.100) for the LDD model are set to $\mu=\gamma$ and $\lambda=\beta / \alpha$. However, such a formal coincidence cannot be justified. The reason is that $\gamma$ and $\lambda$ must not depend on $\alpha$ if the auxiliary quantity $M(\alpha, D)$, as the seed (kernel) function for $S_{\mathrm{F}}^{(\mathrm{LDD})}(D)$, is to satisfy exactly Eq. (9.93). Most importantly, the IMM and LDD models are fundamentally dissimilar because they stem from two different assumptions. The IMM model is based on a clearly defined and quantitatively testable mechanism of enzymatic repair limited by a damage 'fixation' time [55]. On the other hand, the LDD model postulates that a lesion repair is governed by delayed dynamics for the cell radiosensitivity.

\section{Illustrations}

To illustrate the usefulness of the Lambert $W$ functions for a repair-based description of cell surviving fractions, we presently apply two of the discussed models to the 
Experiment and Radiobiological Models for Cell Survival \& Full Effect After Irradiation Cell Surviving Fraction

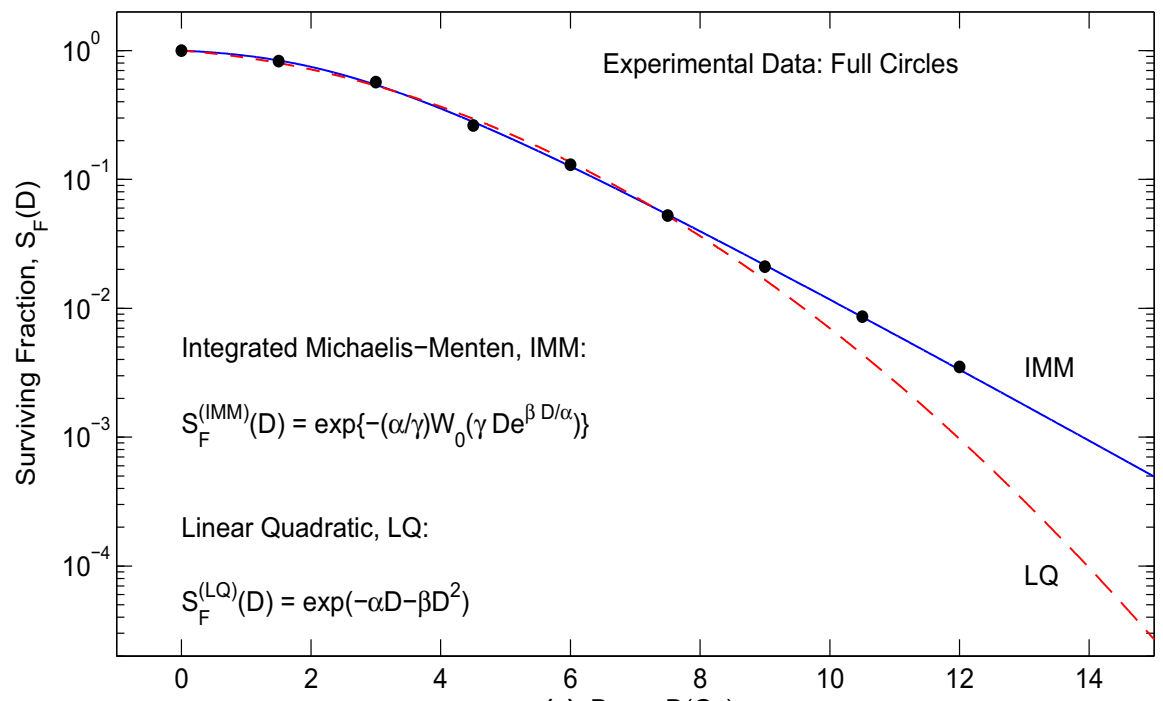

(a) Dose, $D(G y)$

Due to an Account of Repair by Chemical Kinetics, the Integrated Michaelis-Menten Model, IMM, Hugely Outperforms the Linear-Quadratic Model, LQ, for Both Cell Survival and Full-Effect

Full Effect

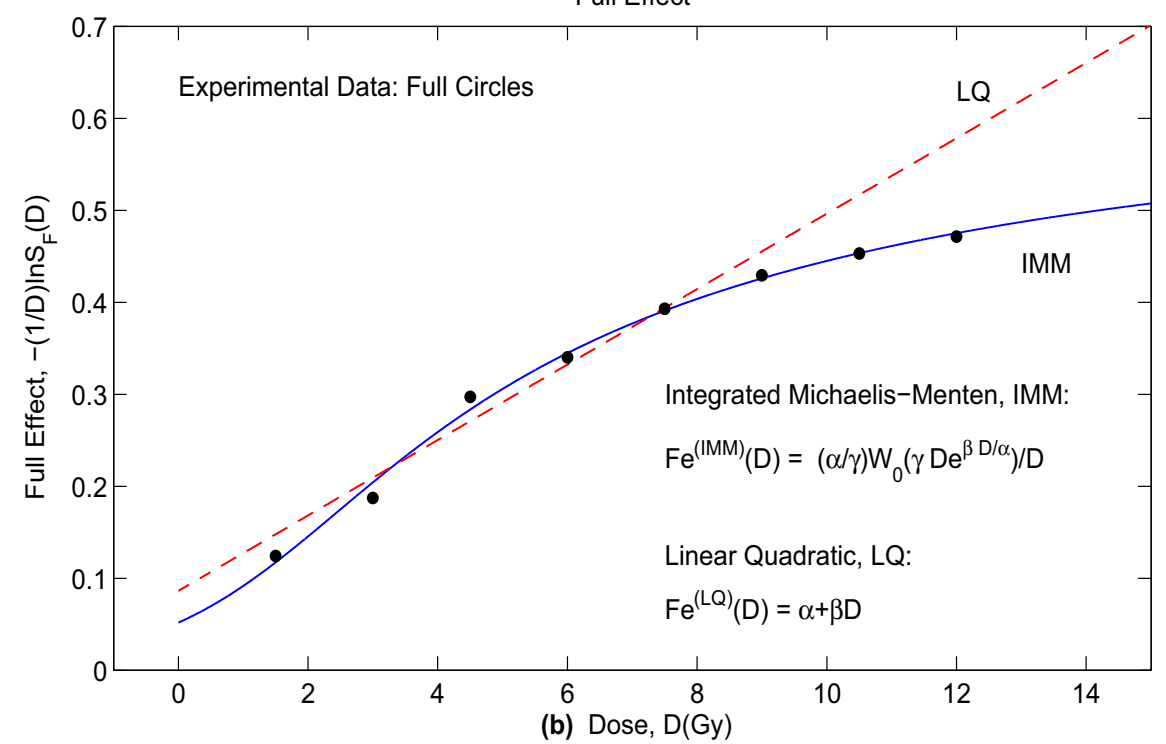

Fig. 1 Cell surviving fraction $S_{\mathrm{F}}(D)$ as a function of radiation instantaneous dose $D$ in Gy (a) and the Fe (Full-effect) plot (b). Experimental data (Chinese hamster V79 cell lines) [122]: full circles. Radiobiological models (present computations): IMM (Integrated-Michaelis-Menten) model: solid curve, and LQ (Linearquadratic) model: dashed curve (see also the rightmost panel '11.5h' in Fig. 1 of Chadwick and Leenhouts [17]) (Color Online) 
Experiment and Radiobiological Models for Cell Survival \& Full Effect After Irradiation Cell Surviving Fraction

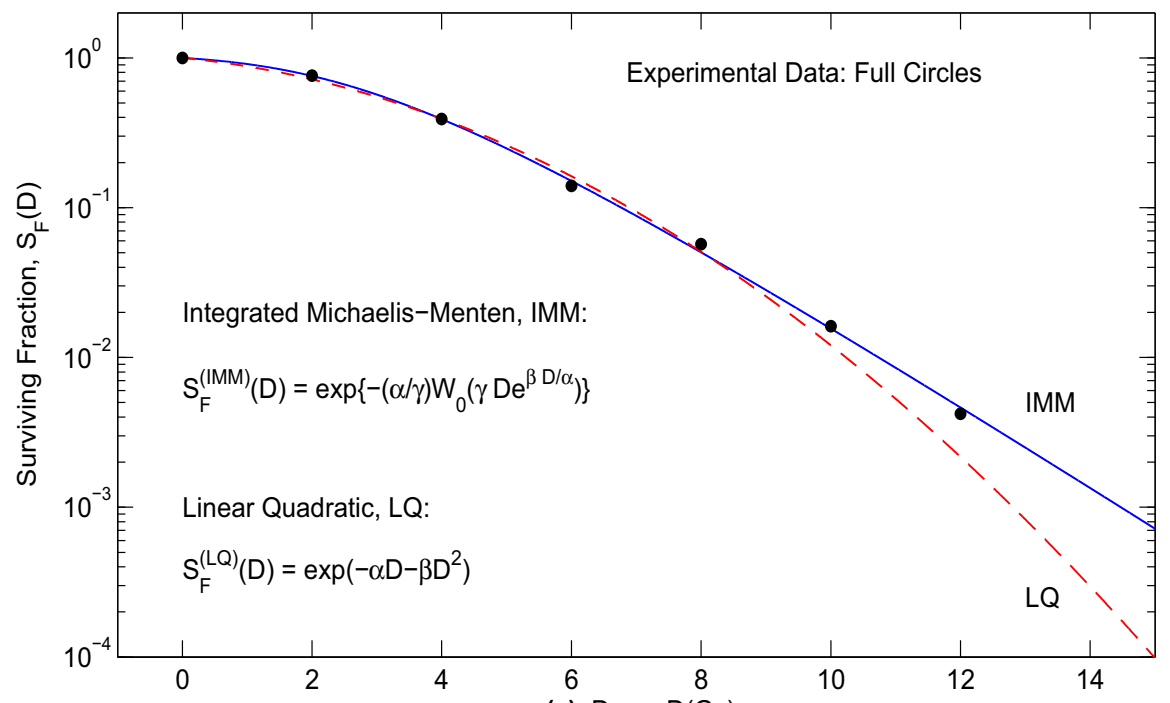

(a) Dose, D(Gy)

Due to an Account of Repair by Chemical Kinetics, the Integrated Michaelis-Menten Model, IMM,

Hugely Outperforms the Linear-Quadratic Model, LQ, for Both Cell Survival and Full-Effect

Full Effect

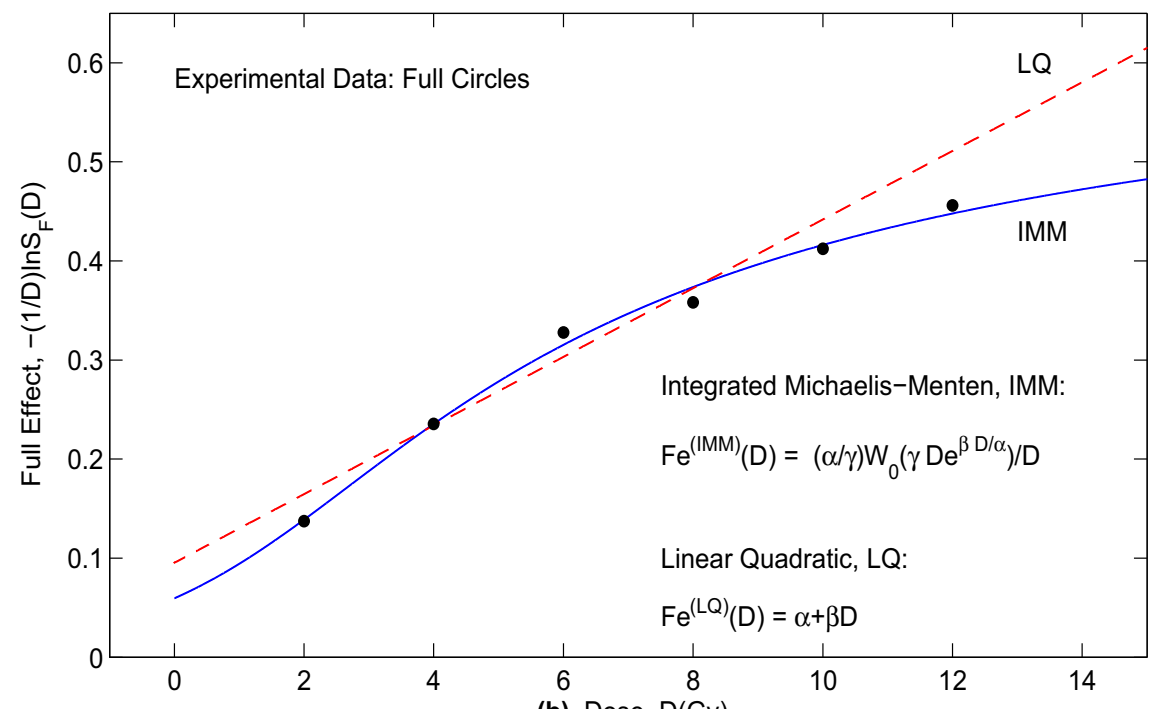

(b) Dose, D(Gy)

Fig. 2 Cell surviving fraction $S_{\mathrm{F}}(D)$ as a function of radiation instantaneous dose $D$ in $\mathrm{Gy}(\mathbf{a})$ and the $\mathrm{Fe}$ (Full-effect) plot (b). Experimental data (Chinese hamster V79 cell lines) [123]: full circles. Radiobiological models (present computations): IMM (Integrated-Michaelis-Menten) model: solid curve and LQ (Linearquadratic) model: dashed curve (Color Online) 
Chinese hamster V79 cells. This is shown in Figs. 1 and 2, where the IMM and LQ models are compared with the available experimental data. Comparisons are made using both cell surviving fractions (Figs. 1a, 2a) and the so-called full-effect curves (Figs. 1b, 2b). The behaviors of the experimental data for cell surviving fractions exhibit three different patterns at low, intermediate and high values of dose $D$. At low and high doses, exponential declines of cell surviving fractions as a function of $D$ are observed with two different decay constants. In between these two asymptotic regions, a curve bending via some shoulders appears. By contrast, the experimental data in the full-effect plot are usually some rising functions of dose $D$.

At all doses, as seen in Figs. 1 and 2, these different trends from the measured data are correctly predicted by the IMM model. Also displayed in Figs. 1a and 2a are the results from the LQ model, which cannot describe the high-dose tails of the measured cell surviving fractions. Moreover, regarding the full-effect plots in Figs. 1b and 2b, the LQ model yields merely the pure straight lines and, thus, fails to detect a plateau at high doses.

Physically, an exponential mode of decline in cell surviving fractions at large $D$ is anticipated. This occurs because the repair system cannot cope with sufficiently high doses that can kill basically all the irradiated cells. Such a phenomenon is also manifested in the full-effect plot, which levels off at sufficiently high doses for which the repair system ceases to function. Namely, for large doses all the available repair molecules (e.g. enzymes in the IMM model or some pool of chemical compounds from the cell environment in the PRL model) are either exhausted (i.e. used up) or incapacitated by the intense radiation.

As has previously been shown for other experimental data [1,2], here too, the corresponding results from the PRL model are close to those of the IMM model. Therefore, to avoid clutter, the cell surviving fractions and the full effect plots from the PRL model are not shown in the present illustrations.

\section{Conclusions}

This review is on the use of the Euler $T$ and Lambert $W$ functions for the radiobiological models based upon chemical kinetics with a special focus on cellular damage repair. Expectedly, experimental data on cell surviving fractions decline exponentially as $\mathrm{e}^{-a D}$ and $\mathrm{e}^{-b D}$ (with two different decay constants $a$ and $b$ ) when the deposited radiation dose $D$ becomes small and large, respectively. A shoulder of a definite width is located in between these two asymptotic regions of exposures to radiations. This shoulder within a limited region of small doses (around $2 \mathrm{~Gy}$ ) has often been approximated by a low-degree polynomial. Such is the situation with the frequently used linear-quadratic model, LQ. This model assumes the Poisson distribution of radiation lesions and gives the cell surviving fraction in terms of the two adjustable parameters $\{\alpha, \beta\}$ via $S_{\mathrm{F}}^{(\mathrm{LQ})}(D)=\exp \left(-\alpha D-\beta D^{2}\right)$. Evidently, the LQ model does not possess the correct high-dose asymptote as $S_{\mathrm{F}}^{(\mathrm{LQ})}(D)$ is reduced to a Gaussian function $\exp \left(-\beta D^{2}\right)$ at large $D$. Further, it has been argued that the LQ model has no mechanistic basis [21]. 
Researchers in this branch of cellular radiobiology (which is relevant to dose planning systems in radiotherapy), usually choose a transition dose $D_{\mathrm{T}}$ to connect a low-dose model (e.g. LQ) with a high-dose, purely exponential inactivation mode of the type $\exp \left(-D / D_{0}\right)$. Here $1 / D_{0}$ is the final slope of the curve for the cell surviving fraction, where $D_{0}$ is the mean lethal dose. Connecting a low- with a high-dose asymptote is customarily made through the Heaviside step function. The ensuing linear-quadraticlinear model, LQL, gives the cell surviving fraction as $S_{\mathrm{F}}^{(\mathrm{LQL})}(D)=S_{\mathrm{F}}^{(\mathrm{LQ})}(D)$ for $D<D_{\mathrm{T}}$ and $S_{\mathrm{F}}^{(\mathrm{LQL})}(D)=S_{\mathrm{F}}^{(\mathrm{LQ})}\left(D_{\mathrm{T}}\right) \mathrm{e}^{-\gamma\left(D-D_{\mathrm{T}}\right)}$ for $D \geq D_{\mathrm{T}}$. Thus, disadvantageously, the LQL has a discontinuous cell survival curve $S_{\mathrm{F}}^{(\mathrm{LQL})}(D)$ and, moreover, it increases the number of the adjustable parameters to four $\left\{\alpha, \beta, \gamma, D_{\mathrm{T}}\right\}$ relative to the LQ model. The LQL model lacks a mechanistic basis. This is inherited from the LQ model, and further exacerbated by artificially imposing the purely exponential cell kill at high doses.

By contrast, as has recently been shown [1,2], these drawbacks of the usual radiobiological models can all simultaneously be lifted. This has been achieved by employing chemical kinetics to derive the mechanistic models that automatically exhibit the correct behaviors at low and high doses, with the adequate predictions of the shoulder at intermediate values of $D$. The corresponding cell survival fractions are given by the explicit, closed expressions that are amenable to the necessary analyses and efficient computations. These models have no need to introduce any transition dose $D_{\mathrm{T}}$ because the passage from one to the other correct exponential tail is smoothly built into the cell surviving fraction by way of the Lambert $W$ functions.

For example, one such model arises from enzyme catalysis in the Michaelis-Menten mechanism, and it is called the integrated Michaelis-Menten model, IMM. The adjective "integrated" means that the standard Michaelis-Menten equation for the reaction velocity (as the rate of concentrations of radiation lesions) is integrated. This integration is carried out analytically giving the biological effect $E_{\mathrm{B}}^{(\mathrm{IMM})}$ in the form of the principal-branch Lambert function $W_{0}$ via $E_{\mathrm{B}}^{(\mathrm{IMM})}=(\alpha / \gamma) W_{0}\left(\gamma D \mathrm{e}^{\beta D / \alpha}\right)$. Assuming the Poisson distribution of lesions, the resulting surviving fraction is $S_{\mathrm{F}}^{(\mathrm{IMM})}(D)=\exp \left\{-(\alpha / \gamma) W_{0}\left(\gamma D \mathrm{e}^{\beta D / \alpha}\right)\right\}$, where $\alpha, \beta$ and $\gamma$ are the kinetic parameters. This survival curve has the required exponential cell kill at low and high doses. Further, it describes cell repair by enzyme catalysis combined with a finite 'fixation' time. Such a type of repair leads to a shoulder in $S_{\mathrm{F}}^{(\mathrm{IMM})}(D)$ at intermediate values of dose $D$.

Chemical kinetics can be used without the assumption that repair is achieved by enzyme catalysis. Instead, some other species from the surrounding pool of the irradiated cells can also be capable of performing repair of the damaged genetic machinery of the cells so that they could resume their cycle with division and reproduction. The system of coupled kinetic differential equations in the Michaelis-Menten formalism cannot be solved exactly in an analytical form, and the quasi-stationary state hypothesis, QSS, is needed to obtain the stated approximate solution [1]. In contradistinction, however, the corresponding system of kinetic differential equations with some pool chemical compounds for cell repair can be solved exactly in an analytical and explicit form without any additional hypothesis [2]. The resulting 
description, called the pool repair Lambert model, PRL, gives the biological effect also in terms of the principal-branch Lambert $W_{0}$ function, but with a different argument, $E_{\mathrm{B}}^{(\mathrm{PRL})}=k_{0} D-p_{0}+(1 / \rho) W_{0}\left(\rho p_{0} \mathrm{e}^{-\rho\left(k_{0} D-p_{0}\right)}\right)$. Again, by invoking the Poisson distribution of radiation lesions, the cell surviving fraction in the PRL model becomes $S_{\mathrm{F}}^{(\mathrm{PRL})}=\exp \left\{-\left(k_{0} D-p_{0}\right)-(1 / \rho) W_{0}\left(\rho p_{0} \mathrm{e}^{-\rho\left(k_{0} D-p_{0}\right)}\right)\right\}$. This cell surviving curve also exhibits the proper limiting behaviors at small as well as large doses, and it correctly describes a shoulder at intermediate doses in accordance with measurements. Here, $p_{0}$ is the initial concentration of the pool molecules, $k_{0}=k_{1}+k_{2}$ and $\rho=k_{2} / k_{1}$, where $k_{1}$ and $k_{2}$ are the rate constants of cell kill and cell repair, respectively. Thus, the three parameters $\left\{p_{0}, k_{0}, \rho\right\}$, or equivalently, $\left\{p_{0}, k_{1}, k_{2}\right\}$ in the PRL model have a clear biological and clinical meaning. They are determined statistically by a minimization procedure using the given input experimental data for dose surviving fractions (or for the full-effect) in the same way as done for the three parameters $\{\alpha, \beta, \gamma\}$ in the IMM model.

Although both the IMM and PRL models are derived from chemical kinetics they, nevertheless, have two different assumptions for explanations of a shoulder at intermediate doses, an exponential tail at high doses, and the entire survival curve as the cell response to radiation insult. In the PRL model, the pool chemical compounds can play a twofold role by being both repair molecules and radiation scavengers. Within the latter role, the pool substances can protect the targeted cells by e.g. donating hydrogens to free radicals produced by radiation. This would prevent free radicals from ionizing DNA, which is the main target. In the PRL model, at high doses, as soon as the pool of repair substances disappears (either by being used up for repair or destroyed by radiation), every subsequent lesion would be lethal, and this amounts to having the mode of the exponential cell kill. Further, at intermediate doses in the PRL model, a larger portion of the irradiated cells would have their damage repaired, and this yields a shoulder in a survival curve.

As to the IMM model, the basic assumption is that lesion repair is carried out by enzymes through enzyme catalysis in the same manner as in any other chemical reaction. In principle, if a sufficient number of enzyme molecules is available, such reactions could continue until the last lesion becomes repaired, as if the repair rate were unlimited. In other words, the Michaelis-Menten mechanism alone would not lead to a shouldered survival curve and, thus, a supplementary assumption is needed. The additional assumption, which limits the repair rate, is that there is a finite time allocated for lesion repair (as supported by measurements). This implies that lethality would occur for all the cells whose lesions have not been repaired between the time of damage and the time of 'fixation' of damage. It is this combination of the limited time for repair, and the enzyme catalysis (with its kinetic parameters) that is responsible for the onset of a shoulder at intermediate doses, and an exponential inactivation in a cell surviving curve at high doses.

In practice, both the IMM and PRL models are capable of fully explaining a variety of experimental data on cell survival after irradiation.

Overall, these advances using chemical kinetics and the Lambert $W$ function offer two mechanistic and clinically interpretable radiobiological models that can advantageously be used in dose planning systems for radiotherapy. This is expected to be 
of help for any level of dose exposure, and thus could hopefully find useful applications especially in stereotactic radiotherapy. This latter radiation therapy deals with localized smaller tumors to which it delivers exclusively high doses (about 10 Gy) per fractions in only a few (about 5) fractions within a week. For dose planning systems in these non-conventional fractionated treatment schedules (the conventional fractionation regimens use about 2 Gy per fraction, 5 days per week, within a month), it is important to rely upon certain adequate, mechanistically-based radiobiological models with the correct behaviors, especially at high doses. The IMM and the PRL models are the two examples of such models that stem from chemical kinetics. The minimization procedures for determining a small number (three) of the mechanistic parameters in the IMM and the PRL models is very efficient. This is the case because the computations of the real principal branch Lambert function $W_{0}$ of real arguments is simultaneously very fast and highly accurate. It is, therefore, hoped that the IMM and PRL radiobiological models will find their way to dose planning systems for both conventional and non-conventional radiotherapy.

Acknowledgements This work is supported by the research grants from Radiumhemmet at the Karolinska University Hospital and the City Council of Stockholm (FoUU) to which the author is grateful.

Open Access This article is distributed under the terms of the Creative Commons Attribution 4.0 International License (http://creativecommons.org/licenses/by/4.0/), which permits unrestricted use, distribution, and reproduction in any medium, provided you give appropriate credit to the original author(s) and the source, provide a link to the Creative Commons license, and indicate if changes were made.

\section{References}

1. Dž Belkić, Repair of irradiated cells by Michaelis-Menten enzyme catalysis: the Lambert function for integrated rate equations in description of surviving fractions. J. Math. Chem. 52, 1253-1291 (2014)

2. Dž Belkić, Survival of radiation-damaged cells via mechanism of repair by pool molecules: the Lambert function as the exact analytical solution of coupled kinetic equations. J. Math. Chem. 52, 1201-1252 (2014)

3. Dž Belkić, K. Belkić, Mechanistic radiobiological models for repair of cellular radiation damage. Adv. Quantum Chem. 70, 43-143 (2015)

4. L. Leksell, The stereotactic method and radiosurgery of the brain. Acta Chirurg. Scand. 102, 316-319 (1951)

5. I. Lax, H. Blomgren, I. Näslund, R. Svanström, Stereotactic radiotherapy of extracranial targets. Z. Med. Phys. 4, 112-113 (1994)

6. A. Hamilton, B. Lulu, H. Fosmire, B. Stea, J.R. Cassadey, Preliminary clinical experience with linear accelerator-based spinal stereotactic radiosurgery. Neurosurgery 36, 311-319 (1995)

7. M. Uematsu, A. Shioda, K. Tahara, T. Fukui, F. Yamamoto, G. Tsumatori, Y. Ozeki, T. Aoki, M. Watanabe, S. Kusano, Focal, high dose and fractionated modified stereotactic radiation therapy for lung carcinoma patients: a preliminary experience. Cancer 82, 1062-1070 (1998)

8. K. Herfarth, J. Debus, F. Lohr, M.L. Bahner, B. Rhein, P. Fritz, A. Höss, W. Schlegel, M.F. Wannenmacher, Stereotactic single-dose radiation therapy of liver tumor: results of a phase I/II trial. J. Clin. Oncol. 19, 164-170 (2001)

9. R. Timmerman, L. Papiez, R. McGarry, Extracranal stereotactic radioablation: results of a phase I study in medically inoperable stage I non-small cell lung cancer. Chest 124, 1946-1955 (2003)

10. R. Timmerman, R. McGarry, C. Yiannoutsos, L. Papiez, K. Tudor, J. DeLuca, M. Ewing, R. Abdulrahman, C. DesRosiers, M. Williams, J. Fletcher, Excessive toxicity when treating central tumors in a phase II study of stereotactic body radiation therapy for medically inoperable early-stage lung cancer. J. Clin. Oncol. 24, 4833-4839 (2006) 
11. R.D. Timmerman, B.D. Kavanagh, L.C. Cho, L. Papies, L. Xing, Stereotactic body radiotherapy in multiple organ sites. J. Clin. Oncol. 25, 947-952 (2007)

12. R. Timmerman, An overview of hypofractionation. Semin. Radiat. Oncol. 18, 215-222 (2008)

13. K.L. Brinkman, B.S. Teh, B. Xu, A review of stereotactic body radiation therapy (SBRT) from the molecular radiobiology perspective. J. Radiat. Oncol. https://doi.org/10.1007/s13566-012-0023-1

14. D.E. Lea, A theory of action of radiations on biological materials: I. The time-intensity factor. Br. J. Radiol. 11, 489-497 (1938)

15. D.E. Lea, Actions of Radiations on Living Cells, 2nd edn. (Cambridge University Press, London, 1955)

16. K.H. Chadwick, H.P. Leenhouts, A molecular theory of cell survival. Phys. Med. Biol. 18, 78-87 (1973)

17. K.H. Chadwick, H.P. Leenhouts, The effect of asynchronous population of cells on the initial slope of dose-effect curves, in Cellular survival after low doses of irradiation, The 6th L.H. Gray Conference Proceedings (Wiley, The Institute of Physics Publishing, Bristol, 1975), pp. 57-63

18. K.H. Chadwick, H.P. Leenhouts, The Molecular Theory of Radiation Biology (Springer, Heidelberg, 1981)

19. J.F. Fowler, The linear-quadratic formula and progress in fractionated radiotherapy. Br. J. Radiol. 62, 679-694 (1989)

20. G.W. Barendsen, Parameters of linear-quadratic radiation dose-effect relationships: dependence on LET and mechanisms of reproductive cell death. Int. J. Radiat. Biol. 7, 1649-55 (1997)

21. M. Zaider, There is no mechanistic basis for the use of the linear-quadratic expression in cellular survival analysis. Med. Phys. 25, 791-792 (1998)

22. K.H. Chadwick, H.P. Leenhouts, Radiation risk is linear with dose at low doses. Br. J. Radiol. 78, 8-10 (2005)

23. N.A.P. Franken, A.L. Oei, H.P. Kok, H.M. Rodermond, P. Sminia, J. Crezee, L.J.A. Stalpers, G.W. Barendsen, Cell survival radiosensitisation: modulation of linear and quadratic parameters of the LQ model (review). Int. J. Oncol. 42, 1501-1515 (2013)

24. J.P. Kirkpatrick, J.J. Meyer, L.B. Marks, The linear-quadratic model is inappropriate to model highdose per fraction effects in radiosurgery. Semin. Radiat. Oncol. 18, 240-243 (2008)

25. J.P. Kirkpatrick, D.J. Brenner, C.G. Orton, The linear-quadratic model is inappropriate to model high-dose per fraction effects in radiosurgery. Semin. Radiat. Oncol. 18, 3381-3384 (2009)

26. M. Guerrero, X.A. Li, Extending the linear-quadratic model for large fraction doses pertinent to stereotactic radiotherapy. Phys. Med. Biol. 49, 4825-4835 (2004)

27. C.S. Park, L. Papiez, R. Timmerman, The unifying (hybrid) survival curve and single fraction equivalent dose: useful tools in understanding the potency of ablative radiation therapy. Int. J. Radiat. Oncol. Biol. Phys. 69(Supplement 1), 623-624 (2007)

28. C. Park, L. Papiez, S. Zhang, M. Story, R.D. Timmerman, Universal survival curve and single fraction equivalent dose: Useful tools in understanding potency of ablative radiotherapy. Int. J. Radiat. Oncol. Biol. Phys. 70, 847-852 (2008)

29. M. Astrahan, Some implications of linear-quadratic-linear radiation dose-response with regard to hypofractionation. Med. Phys. 35, 4161-4172 (2008)

30. M. Astrahan, BED Calculations for fractions of very high dose: in regard to Park et al. (Int. J. Radiat. Oncol. Biol. Phys. 2007; 69: S623-S624). Int. J. Radiat. Oncol. Biol. Phys. 71, 963 (2008)

31. C. Park, R.D. Timmerman, In response to Dr. Astrahan. Int. J. Radiat. Oncol. Biol. Phys. 71, 963-964 (2008)

32. B.D. Kavanagh, F. Newman, Toward a universal survival curve. Int. J. Radiat. Oncol. Biol. Phys. 71, 958-959 (2008)

33. W.A. Tome, Universal survival curve and single fraction equivalent dose: useful tools in understanding potency of ablative radiotherapy: in regard to Park, et al. Int. J. Radiat. Oncol. Biol. Phys. 72, 1620 (2008)

34. F. McKenna, S. Ahmad, Toward a universal survival curve. Int. J. Radiat. Oncol. Biol. Phys. 73, 640-640 (2008)

35. F. McKenna, S. Ahmad, Fitting techniques of cell survival curves in high-dose region for use in stereotactic body radiation therapy. Phys. Med. Biol. 54, 1593-1608 (2009)

36. C. Park, L. Papiez, R.D. Timmerman, In reply to Drs. McKenna and Ahmad. Int. J. Radiat. Oncol. Biol. Phys. 73, 641-641 (2009) 
37. O. Hug, A.M. Kellerer, Zur interpretation der dosiswirkungsbeziehungen in der strahlenbiologie. Biophysik 1, 20-32 (1963)

38. R.H. Haynes, The interpretation of microbial inactivation and recovery phenomena. Radiat. Res. (Supplement) 6, S1-S29 (1966)

39. A. Sánchez-Reyes, A simple model of radiation action in cells based on repair saturation mechanism. Radiat. Res. 130, 139-147 (1992)

40. A. Sánchez-Reyes, B. Farrús, A. Biete, A new theoretical formula for fractionated radiotherapy based on a saturable cellular repair mechanism. Acta Oncol. 32, 57-62 (1993)

41. K.E. Ekstrand, The Hug-Kellerer equation as the universal cell surviving curve. Phys. Med. Biol. (Notes) 55, N267-N273 (2010)

42. S.J. González, G.A.S. Cruz, The photon-isoeffective dose in boron neutron capture therapy. Radiat. Res. 178, 609-621 (2012)

43. R.O. Fabias, S. Bortolussi, Pablo R. Menéndez, S.J. González, Exploring boron neutron capture therapy for non-small cell lung cancer. Phys. Med. 30, 888-897 (2014)

44. C.A. Tobias, E.A. Blakely, E.Q.H. Ngo, T.C.H. Yang, The repair-misrepair model of cell survival, in Radiation Biology in Cancer Research, ed. by R.R. Meyn, H.R. Withers (Raven Press, New York, 1980), pp. 195-230

45. S.B. Curtis, Lethal and potential lethal lesions induced by radiation-a unified repair model. Radiat. Res. 10, 252-270 (1987)

46. J. Kiefer, A repair fixation model based on classical enzyme kinetics, in Quantitative Mathematical Models in Radiation Biology, ed. by J. Kiefer (Springer, Berlin, 1988), pp. 171-179

47. Dž Belkić, Parametric analysis of time signals and spectra from the perspective of quantum physics and chemistry. Adv. Quantum Chem. 61, 145-260 (2011)

48. Dž Belkić, K. Belkić, High-resolution signal processing in magnetic resonance spectroscopy for early cancer diagnostics. Adv. Quantum Chem. 62, 345-347 (2011)

49. Dž Belkić, K. Belkić, Padé-Froissart exact signal-noise separation in nuclear magnetic resonance spectroscopy. J. Phys. B Atom. Mol. Opt. Phys. 44, 125003 (2011)

50. Dž Belkić, K. Belkić, Mechanistic repair-based Padé linear-quadratic model for cell response to radiation damage. Adv. Quantum Chem. 65, 407-449 (2013)

51. Dž Belkić, K. Belkić, Mechanistic description of survival of irradiated cells: repair kinetics in Padé linear-quadratic or differential Michaelis-Menten model. J. Math. Chem. 51, 2572-2607 (2013)

52. Dž Belkić, K. Belkić, Molecular imaging in the framework of personalized cancer medicine. Isr. Med. Assoc. J. 15, 665-672 (2013)

53. B. Andisheh, M. Edgren, Dž Belkić, P. Mavroidis, A. Brahme, B.K. Lind, A comparative analysis of radiobiological models for cell surviving fractions at high doses. Techn. Cancer Res. Treat. 12, 183-192 (2013)

54. J. Calkins, A method for analysis of radiation response based on enzyme kinetics. Radiat. Res. 45, 50-62 (1971)

55. J. Calkins, Alternative repair models capable of generating shouldered dose-response curves. Int. J. Radiat. Biol. 59, 997-999 (1991)

56. J. Laurie, J.S. Orr, C.J. Foster, Repair processes and cell survival. Brit. J. Radiol. 45, 362-368 (1972)

57. D.T. Goodhead, Saturable repair models of radiation action in mammalian cells. Radiat. Res. (Supplement) 104, 58-67 (1985)

58. J.M. Nelson, L.A. Braby, N.F. Metting, W.C. Roesch, Interpreting survival observations using phenomenological models, in Quantitative Mathematical Models in Radiation Biology, ed. by J. Kiefer (Springer, Berlin, 1988), pp. 125-134

59. J.H. Lambert, Observationes varie in mathesin puram. Acta Helvetica, Physico-mathematicoanatomico-botanico-medica, Basel, 3, 128-168 (1758). http://www.kuttaka.org/ JHL/L1758c.pdf

60. J.H. Lambert, Observationes analitiques. Nouveaux mémoires de l'académie royale des sciences et belle-lettres, Berlin, 1 (1770)

61. L. Euler, Deformulis exponentialibus replicatis. Reprinted in: Leonahard Euleri Opera Omnia, Ser. Prima. Opera Math. 15, 268-297 (1927). (original publication year: 1777)

62. L. Euler, De serie Lambertina plurimisque eius insignibus proprietatibus. Acta Acad. Scient. Petropol. 2, 29-51 (1783) (Reprinted in: L. Euler, Opera Omnia, Series Prima, Leipzig, Germany, Commentationes Algebraicae 6, 350-369 (1921); http://math.dartmouth.edu/ euler.docs/originals/E532.pdf)

63. E.M. Wright, The linear difference-differential equation with constant coefficients. Proc. R. Soc. Edinb. A Math. Phys. Sci. 62, 387-393 (1949) 
64. E.M. Wright, A non-linear difference-differential equation. J. Reine Angew. Math. 194, 66-87 (1955)

65. E.M. Wright, Solution of the equation $z \mathrm{e}^{z}=a$. Proc. R. Soc. Edinb. A Math. Phys. Sci. 65, 193-203 (1959)

66. C.E. Siewert, E.E. Burniston, An exact analytical solution of Kepler's equation. Celest. Mech. 6, 294-304 (1972)

67. E.E. Burniston, C.E. Siewert, The use of Riemann problems in solving a class of transcendental equations. Proc. Camb. Philos. Soc. 73, 111-118 (1973)

68. C.E. Siewert, An exact analytical solution of an elementary critical condition. Nucl. Sci. Eng. 51, 78-79 (1973)

69. C.E. Siewert, E.E. Burniston, On a critical condition. Nucl. Sci. Eng. 52, 150-151 (1973)

70. E.E. Burniston, C.E. Siewert, Exact analytical solution of the transcendental equation $a \sin \zeta=\zeta$. SIAM J. Appl. Math. 4, 460-465 (1973)

71. C.E. Siewert, C. Essig, An exact solution of a molecular field equation in the theory of ferromagnetism. J. Appl. Math. Phys. 24, 281-286 (1973)

72. C.E. Siewert, E.E. Burniston, Exact analytical solution of $z \mathrm{e}^{z}=a$. J. Math. Anal. Appl. 73, 626-632 (1973)

73. C.E. Siewert, A.R. Burkart, On double zeros of $x=\tanh (a x+b)$. J. Appl. Math. 24, 435-439 (1973)

74. C.E. Siewert, Solutions of the equation $z \mathrm{e}^{z}=a(z+b)$. J. Math. Anal. Appl. 46, 329-337 (1974)

75. C.E. Siewert, E.E. Burniston, An exact analytical solution for the position-time relationship for an inverse-distance-squared force. Int. J. Eng. Sci. 12, 861-863 (1974)

76. C.E. Siewert, E.E. Burniston, An exact analytical solution of $x \operatorname{coth} x=\alpha x^{2}+1$. J. Comput. Appl. Math. 2, 19-26 (1976)

77. C.E. Siewert, Explicit results for the quantum-mechanical energy states basic to a finite square-well potential. J. Math. Phys. 19, 434-435 (1978)

78. C.E. Siewert, J.S. Phelps III, On solutions of a transcendental equation basic to the theory of vibrating plates. SIAM J. Math. Anal. 10, 105-108 (1979)

79. C.E. Siewert, J.S. Phelps, Explicit solutions of $a \tan (\xi k \pi)+\tanh \xi=0$. J. Comp. Appl. Math. 5, 99-103 (1979)

80. R.M. Corless, G.H. Gonnet, D.E.G. Hare, D.J. Jeffrey, Lambert's $W$ function in Maple. Maple Techn. Newslett. 9, 12-22 (1993)

81. D.J. Jeffrey, R.M. Corless, D.E.G. Hare, D.E. Knuth, Sur l'inversion de $y^{\alpha} \mathrm{e}^{y}$ au moyen de nombres de Stirling associés. C. R. Acad. Sci. Paris Série I 320, 1449-1452 (1995)

82. D.J. Jeffrey, D.E.G. Hare, R.M. Corless, Unwinding the branches of the Lambert function. Math. Sci. 21, 1-7 (1996)

83. R.M. Corless, G.H. Gonnet, D.E.G. Hare, D.J. Jeffrey, D.E. Knuth, On the Lambert $W$ function. Adv. Comput. Math. 5, 329-359 (1996)

84. R.M. Corless, D.J. Jeffrey, D.E. Knuth, A sequence of series for the Lambert $W$ function, in Proceedings of International Symposium on Symbolic and Algebra Computation (ACM Press, Maui, Hawaii, USA, Ed. W.W. Kuechlin), pp. 197-204 (1997). https://doi.org/10.1145/258726.258783

85. J.M. Borwein, R.M. Corless, Emerging tools for experimental mathematics. Am. Math. Month. 106, 889-909 (1999)

86. T.C. Scott, J.F. Babb, A. Dalgarno, J.D. Morgan III, Resolution of a paradox in the calculation of exchange forces for $\mathrm{H}_{2}^{+}$. Chem. Phys. Lett. 203, 175-183 (1993)

87. T.C. Scott, J.F. Babb, A. Dalgarno, J.D. Morgan III, Calculation of exchange forces: general results and specific models. J. Chem. Phys. 99, 2841-2854 (1993)

88. S. Schnell, C. Mendoza, Closed form solution for time-dependent enzyme kinetics. J. Theor. Biol. 187, 202-212 (1997)

89. C.T. Goudar, J.R. Sonnad, R.G. Duggleby, Parameter estimation using a direct solution of the integrated Michaelis-Menten equation. Biochim. Biophys. Acta 1429, 377-383 (1999)

90. C. Katsimpiri, P.E. Nastou, P.M. Pardalos, Y.C. Stamation, The ubiquitous Lambert $W$ function and its classes in sciences and engineering, in Contributions in Mathematics and Engineering in Honor of Constantin Carathéodory, ed. by P.M. Pardalos, T.M. Rassias (Springer, Berlin, 2016), pp. 323-342

91. V. Barsan, Siewert solutions of the transcendental equations, generalized Lambert functions and physical applications. Open Phys. 16, 232-242 (2018). arXiv:1703.10052v1 [physics.gen-ph] (2017)

92. Int. Workshop, Celebrating 20 years of the Lambert $W$ function. Western University, London, Ontario, Canada (July 25-28, 2016). www.apmaths.uwo.ca/ djeffrey/LambertW/LambertW.html 
93. F.N. Fritsch, R.E. Shafer, W.P. Crowley, Algorithm 443: solution of the transcendental equation $w \mathrm{e}^{w}=x$. Commun. ACM 16, 123-124 (1973)

94. D.A. Barry, S.J. Barry, P.J. Culligan-Hensley, Algorithm 743: WAPR: a FORTRAN routine for calculating real values of the W-function. Assoc. Comput. Mach. Trans. Math. Softw. 21, 172-181 (1995)

95. D.H. Bailey, Y. Hida, X.S. Li, B. Thompson, Arprec: an arbitrary precision computation package (2002). http://crd.lbl.gov/ dhbailey/dhbpapers/arprec.pdf; http://crd.lbl.gov// dhbailey/mpdist

96. D.H. Bailey, High-precision floating-point arithmetic in scientific computation. Comput. Sci. Eng. 7, 54-61 (2005)

97. W. Gautschi, The Lambert $W$-functions and some of their integrals: a case study for high-precision computations. Numer. Algorithm 57, 27-34 (2011)

98. W. Gautschi, Matlab open source codes from Ref. [97]. http://www.cs.purdue.edu/archives/2002/ wxg/codes/LAMBERTW.html

99. D. Veberič, Lambert $W$ function for applications in physics. Comput. Phys. Comm. 183, 2622-2628 (2012) (open cource in C++, http://cpc.cs.qub.ac.uk/summaries/AENC_v1_0.html)

100. Z.L. Krougly, D.J. Jeffrey, Implementation and application of extended precision in Matlab. Math. Meth. Appl. Comput., in Proceedings of the 11th International Conference on Mathematical Methods and Computational Techniques in Electrical Engineering (Athens, 28-30 September, 2015), pp. 103108

101. F. Johansson, Computing the Lambert $W$ function in arbitrary-precision complex interval arithmetic. HAL (Arhives-Ouvertes.fr) (2017). http://hal.inria.fr/hal-01519823 (2017); http://arblib.org/; arXiv:1705.03266v1 [cs.MS]; https://arxiv.org/pdf/1705.03266.pdf

102. A. Adler, Lambert $W$-function (lam $W$-package, lambertW) (2017). https://bitbucket.org/aadler/ lamw

103. Dž Belkić, All the trinomial roots, their arbitrary powers and logarithms: from the perspective of the Lambert $W$ function, the complete Bell polynomials $B_{n}$ and the Fox-White $\Psi$ function. J. Math. Chem. (to be submitted shortly, 2018)

104. W.E. Ricker, Stock and recruitment. J. Fish. Res. Board Can. 11, 559-623 (1954)

105. I.S. Gradsteyn, L.M. Ryzhik, in Table of Integrals, Series and Products, 6th edn., ed. by A. Jeffrey, D. Zwillinger (Academic Press, New York, 2000)

106. R.P. Stanley, Enumerative Combinatorics, vol. 1 (Cambridge University Press, Cambridge, 1999)

107. R.P. Stanley, Enumerative Combinatorics, vol. 2 (Cambridge University Press, Cambridge, 2001)

108. F. Chapeau-Blondeau, A. Monir, Numerical evaluation of the Lambert $W$ function and application to generation of generalized Gausssian noise with exponent 1/2. IEEE Trans. Signal Process. 50, 2160-2165 (2002)

109. D.A. Barry, L. Li, D.-S. Jeng, Comments on numerical evaluation of the Lambert $W$ function and application to generation of generalized Gausssian noise with exponent 1/2. IEEE Trans. Signal Process. 52, 1456-1458 (2004)

110. N.D. Hayes, The roots of the equation $x=(c \exp )^{n} x$ and the cycles of the substitution $\left(x \mid c \mathrm{e}^{x}\right),$. Q. J. Math. Oxf. 3, 81-90 (1952)

111. Dž Belkić, Quantum-Mechanical Signal Processing and Spectral Analysis (Taylor \& Francis, London, 2005)

112. L. Michaelis, M.L. Menten, Die kinetik der invertinwirkung. Biochem. Z. 49, 333-369 (1913) [English translation by R.S. Goody and K.A. Johnson, The kinetics of invertase action. Biochem. 50, 82648269 (2011); Supporting Information: The full text (34 pp) of the German to English translation of the original paper by Michaelis and Menten (1913, op. cit.) available at: http://pubs.acs.org]

113. G.E. Briggs, J.B.S. Haldane, A note on the kinetics of enzyme action. Biochem. J. 19, 338-339 (1925)

114. D.D. van Slyke, G.E. Cullen, The mode of action of urease and of enzymes in general. J. Biol. Chem. 19, 141-180 (1914)

115. E.L. Powers, Consideration of survival curves and target theory. Phys. Med. Biol. 7, 3-28 (1962)

116. J.S. Orr, C.S. Hope, S.E. Wakerley, A metabolic theory of cell survival curves. Phys. Med. Biol. 11, 103-108 (1966)

117. J.F. Malone, The kinetics of recovery of cellular proliferation after irradiation. PhD Thesis (unpublished), The National University of Ireland, Dublin, 1972

118. J.S. Orr, J. Laurie, J. Kirk, J.F. Malone, The "pool" and the initial slope of survival curves for high- and low-LET radiation, in Cellular survival after low doses of irradiation, The 6th L.H. Gray Conference Proceedings (Wiley, The Institute of Physics Publishing, Bristol, 1975), pp. 86-87 
119. J.F. Malone, I.A. Kinsella, J.H. Hendry, Prediction of the initial shape of survival curves when direct measurement is not possible, in Cellular survival after low doses of irradiation, The 6th L.H. Gray Conference Proceedings (Wiley, The Institute of Physics Publishing, Bristol, 1975), pp. 313-318

120. E.L. Powers, Responses of cells to radiation sensitizers: methods of analysis. Int. J. Radiat. Biol. 42, 629-651 (1982)

121. J.W. Müller, Deux nouvelles expressions concernant un temps mort cumulatif. BIPM Working Party Note 217, 5 (1980). www.bipm.org/en/publications/wpn.html; BIPM: Bureau Internationale des Poids et Mesures (International Bureau of Weights and Measures)

122. W.K. Sinclair, Protection against lethal X-ray damage during the cell cycle of Chinese humster cells. Radiat. Res. 39, 135-154 (1969)

123. Y. Shibamoto, S. Otsuka, H. Iwata, C. Sugie, H. Ogino, N. Tomita, Radiobiological evaluation of the radiation dose as used in high-precision radiotherapy: effect of prolonged delivery time. J. Radiat. Res. 53, 1-9 (2012)

\section{Affiliations}

\section{Dževad Belkićc ${ }^{1,2}$}

$凶$ Dževad Belkić

Dzevad.Belkic@ki.se

1 Department of Oncology-Pathology, Nobel Medical Institute - Karolinska Institute, P.O. Box 260, 171 76, Stockholm, Sweden

2 Medical Radiation Physics and Nuclear Medicine, Karolinska University Hospital, P.O. Box 260, 17176 , Stockholm, Sweden 